\title{
Learning Analytics para una visión tipificada del aprendizaje de los estudiantes. Un estudio de caso
}

\author{
$\mathrm{M}^{\mathrm{a}}$ Covadonga de la Iglesia Villasol (10 @ \\ Universidad Complutense de Madrid (UCM), España.
}

Resumen. Los nuevos métodos de enseñanza-aprendizaje, con apoyo de los entornos virtuales, como extensión asincrónica de las aulas, han determinado que el docente asuma nuevos roles, algunos de los cuales pasan por aumentar su capacidad de observación y convertirse en analista del proceso de aprendizaje de los estudiantes, del uso que hacen de las plataformas digitales, su interrelación con ellas y entre sí, y de cómo adquieren conocimientos y desarrollan algunas competencias. Desde ésta óptica, el trabajo aborda una primera explotación de los usos y registros estadísticos, que a modo de huella digital, dejan los estudiantes en cursos presenciales en los espacios virtuales, y que definen tipologías y patrones diferenciados de aprendizaje según ritmos, aspecto éste que puede conllevar a una reflexión y reorientación del proceso de enseñanza-aprendizaje futuro. En concreto, el estudio de caso recoge resultados descriptivos de la interacción del uso que los estudiantes realizan de los recursos digitales con la evaluación continua y las calificaciones en la asignatura de Análisis Microeconómico Avanzado, del grado en Economía (GECO), UCM en el curso 2018-19, aportando un perfil de uso aún muy tradicional y polarizado, vinculada con mínimos desfases al cronograma de las actividades propuestas por el profesor.

Palabras clave: registros de usos; estudiantes; aprendizaje, gamificación; competencias; learning analytics.

Learning Analytics para uma visão tipificada da aprendizagem dos estudantes. Um
estudo de caso
Resumo. Os novos métodos de ensino-aprendizagem, com o apoio dos ambientes vir-
tuais como extensão assíncrona da sala de aula, determinaram que o professor assuma
novos papéis, alguns dos quais vão desde aumentar a sua capacidade de observação
até o de atuar como um analista do processo de aprendizagem dos estudantes, do uso
de plataformas digitais e sua inter-relação com elas e entre elas, e de como adquirem
conhecimento e desenvolvem algumas competências. A partir desta perspectiva, o
trabalho aborda uma primeira exploração de usos e registros estatísticos, que, no
tocante à marca digital, deixam os estudantes de cursos presenciais nos espaços
virtuais e que definem tipologias e padrões diferenciados de aprendizagem de acordo
com os ritmos, um aspecto que pode levar a uma reflexão e reorientação do futuro
processo de ensino-aprendizagem. Especificamente, o estudo de caso inclui resultados
descritivos da interação do uso de recursos digitais por estudantes com a avaliação
contínua e o resultado obtidos na disciplina de Análise Microeconômica Avançada do
curso de graduação em Economia (GECO), de 2018-19 da UCM, contribuindo com
um perfil de uso ainda muito tradicionale polarizado, vinculado a defasagens mínimas
no cronograma de atividades proposto pelo professor. Keywords: registros de usos; estudantes; aprendizagem; gamificação; competências; learning analytics.

Learning Analytics for a typified vision of student learning. A case study

Abstract. The new methods of teaching and learning, based on virtual environments, which are an asynchronous extension of the classrooms, has determined that the teacher has had to assume new roles, some of which go through to increase their observation capacity and become an analyst of the learning process that the students follow, about the uses they make of digital platforms, how they interrelate with them and with others students, and how they acquire knowledge and develop skills and competences. From this point of view, the work deals with the description of statistical uses and registers, which, as a fingerprint, leave students in face-to-face courses in the virtual platforms, and which can define typologies and patterns differentiated learning, aspect that can lead to a reflection and reorientation of the future teaching-learning process. Specifi- 
cally, the case study includes descriptive results of the interaction of students' use of digital resources with continuous assessment and grades in the course of Advanced Microeconomic Analysis, of the degree in Economics (GECO), at the UCM in the 201819 academic year, providing a profile of use that is still very traditional and polarized, linked to minimum lags in the timetable of the proposed activities by the teacher.

Keywords: records of uses, students, learning, gamifying, skills, learning analytics.

\section{Introducción y justificación}

El Espacio Europeo de Educación Superior (EEES) reorientó el foco de atención del sistema educativo español hacia la implantación del aprendizaje centrado en el estudiante, facilitando que sea activo, autónomo, crítico y reflexivo (Herrero, 2014), para lo cual han sido múltiples los entornos de aprendizaje instaurados y que coexisten con total naturalidad, con el objetivo final de que los estudiantes adquieran necesariamente los conocimientos específicos de su titulación, y desarrollen un corpus competencial requerido en el entorno profesional.

Este trabajo recoge el análisis cualitativo de la explotación de las huellas, registros o rastros que los estudiantes dejan, sin que pueda ser de otra forma, tanto en el uso del aula virtual como de otras plataformas digitales en abierto ${ }^{1}$, disponibles como un complemento indispensable e integrado de forma armónica con la enseñanza presencial, a los que los estudiantes acceden con ritmos distintos y picos en fechas puntuales (previas a pruebas de evaluación, o de forma continuada y secuencial con el progreso del curso). Como a los estudiantes universitarios se les presupone un desarrollo de competencias digitales ${ }^{2}$, deben ser en cierto grado autónomos para seleccionar y gestionar la información más útil para su progreso en el aprendizaje, así como para prefijar sus ritmos de trabajo fuera del aula, con independencia de las recomendaciones y el calendario prefijado por el profesor.

El docente que complementa el aula presencial con entornos virtuales y busca fomentar ese aprendizaje activo, según la naturaleza de la materia a impartir, las actividades y las dinámicas de aprendizaje realizadas, puede segmentar al grupo en subgrupos formados por estudiantes con personali-

${ }^{1}$ Algunos materiales han sido elaborados para diversos proyectos de innovación y mejora de la calidad docente, la UCM.

2 El Real Decreto 1105/2014, de 26 de diciembre, vigente en el curso 2001819 , establece el currículo básico de la Educación Secundaria Obligatoria y del Bachillerato (BOE Núm. 3, 3 de enero de 2015 Sec. I. Pág. 169 y ss), e indica como competencias del currículo la Comunicación lingüística (lengua materna y extranjeras); la Competencia matemática y competencias básicas en ciencia y tecnología; la Competencia digital; Aprender a aprender; Competencias sociales y cívicas; Sentido de iniciativa y espíritu emprendedor; y la Conciencia y expresiones culturales. 
dades, tipos de inteligencias, formas y ritmos de aprendizaje diversos, a los que suele observar durante el curso y preevaluar. Pero sería diferenciador disponer de prediseños anticipados, ex ante, hoy por hoy casi impensable ${ }^{3}$, sobre cómo aprenden los estudiantes que sirvieran de guía al profesor en el diseño de un desarrollo con tintes autónomos, y que suelen ser sustituidos por el uso de ciertos marcadores secuenciales del mismo (Cano, 2009). Así, el docente observa con continuidad el progreso (ritmos) de los estudiantes en la adquisición de conocimientos, el avance en los usos de los recursos tecnológicos, así como las huellas que dejan en su interrelación con los entornos digitales al transitar por las tareas individuales y/o colectivas, y ésta es la información de la que se alimenta el estudio de caso.

El rastro que queda al acceder a las herramientas, carpetas, actividades, links, wikis, foros, etc. de las plataformas digitales, es un banco de datos que está siendo objeto de explotación en la mayor parte de los Centros Educativos y Universidades y que en menos de dos décadas ha generado una creciente y fructífera literatura empírica, Learning Analytics ${ }^{4}$ ( $L A$, analítica del aprendizaje), con numerosos usos y aplicaciones, entre los que destacan la posibilidad de que el docente conozca cómo asincrónicamente trabajan los estudiantes, ritmos, frecuencias, franjas horarias, repetición de accesos, tiempos de usos, opciones fallidas, etc. (Vela-Pérez, Hernández-Estrada, Tirado y otros, 2017) y en su caso, si pudiera conocerlos a tiempo, tener la opción de reorientarles para una mayor eficiencia o reelaborar una estrategia de aprendizaje diferenciada o personalizada. Más limitados son los trabajos en los que la analítica de los registros de datos persigue la observación y estudio particularizado al desarrollo de ciertas competencias, como pueda ser el trabajo en grupo (Guitert, Romeu y Pérez, 2007), o el estudio colaborativo (Martínez, Arteaga y Pérez, 2017) o la gestión eficaz del tiempo o el desarrollo de técnicas de negociación, por ejemplo, siendo compleja su evaluación y análisis descriptivo, al requerir de marcadores o indicadores óptimos y validados en diversas dimensiones, no siempre disponibles, definidos parcialmente o con deficiencias.

${ }^{3}$ La información, en general, se analiza a posteriori y no en tiempo real, lo cual requeriría algoritmos de aplicación secuencial.

${ }^{4}$ La definición oficial de Learning Analytics se realiza en la First international Conference on Learning Analytics and Knowledge (LAK 2011), adoptada por la Society for Learning Analytics Research (SoLAR), como la medición, recopilación, análisis e informe de datos sobre los alumnos y sus contextos, con el fin de comprender y optimizar el aprendizaje y los entornos en los que se produce. 


\section{Algunos elementos del estado de la cuestión}

Dos aspectos del marco teórico concentran el interés que contextualiza el trabajo, como son los nuevos roles que el docente tiene que adquirir en la era digital y cómo gestionar la masiva información registrada a partir de los usos que los estudiantes realizan en las plataformas de aprendizaje digitales, así como definir instrumentos cuantitativos para dicho fin. Obviamente la revisión de la literatura planeará sobre ambos elementos, que si bien pueden ser apasionantes en sí mismos, no va a ser exhaustiva, por cuanto hacerlo excede los objetivos de este trabajo empírico.

El docente en la era digital ha asumido como roles (Iglesia, 2006 y 2018), ser un diseñador de nuevos entornos de aprendizaje, que combina diversos recursos y técnicas educativas, convertido en un generador y evaluador de recursos útiles para el autoaprendizaje, con el doble objetivo de transmitir conocimiento técnico, especializado y favorecer en ese aprendizaje el desarrollo de competencias requeridas en el entorno profesional, sin dejar de ser orientador, tutor, motivador, a la vez que un co-aprendiz de su profesión, y por tanto, un acompañante en el proceso formativo. Así, si la figura del docente adopta nuevos roles y adquiere una nueva perspectiva, el alumno ha dejado de ser un mero receptor pasivo de contenidos, convirtiéndose en el actor principal de su aprendizaje (Díaz-Barriga, 2011).

Con esta visión radial, Harrison y Killion (2007), indican que será un facilitador del éxito en el aprendizaje de los estudiantes, especialista curricular, un proveedor de recursos, instructor, facilitador de apoyo en el aula y del aprendizaje, mentor de sus estudiantes, analista de datos, catalizador del cambio, líder de equipos y aprendiz. Prensky (2013) señaliza el rol de competente digital ${ }^{5}$, y su incidencia en tres grandes rúbricas como son el de entrenador (por la observación individual y motivación al estudiante), guía (ayuda a conseguir los objetivos) y de experto en la instrucción (a través del conocimiento y la creatividad), con el objetivo de conseguir un efectivo, motivador y atractivo aprendizaje, para lo cual utiliza la tecnología disponible para mejorar la metodología del proceso de enseñanza-aprendizaje (Bates, 2015). Para Viñals y Cuenca (2016), los nuevos roles pasan por convertirse en un organizador, guía, generador, acompañante, coacher, gestor de aprendizaje,

${ }^{5}$ El informe A Framework for Developing and Understanding Digital Competence in Europe (Ferrari, 2013), indica que se es competente digital si se cubren las dimensiones referidas a la búsqueda y gestión de la información digital; comunicar e interactuar; crear contenido multiformato y multimedia; identificar y resolver problemas, y gestionar la protección y seguridad digital de la información. https://bit.ly/23osQI7 
orientador, facilitador, tutor, dinamizador o asesor, y señalan que cambian la transmisión unidireccional del conocimiento por el intercambio horizontal de información.

Consecuentemente, el espacio físico en el que se desarrolla el proceso de enseñanza aprendizaje adquiere relevancia, por cuanto que el aula física deja de ser el único escenario de aprendizaje, complementado por espacios reales y virtuales, escogidos o diseñados para una multiplicidad asincrónica. Este multiespacio de aprendizaje se vincula a un trabajo activo y colaborativo entre los alumnos y de éstos con el profesor, aprovechando las herramientas tecnológicas y las metodologías innovadoras (Gutiérrez, Palacios y Torrego, 2010).

Con ésta descripción de roles, el profesor como observador directo del aprendizaje de sus estudiantes, empieza a adoptar entre sus tareas la gestión y el análisis de la información, que genera su actividad y la de sus estudiantes (convertido en el analista de datos a que se refiere Harrison y Killion (2007), que le permite conocer mejor su propia acción docente, la tipología de sus estudiantes y los resultados, así como las actitudes y compromiso que adquieren con el programa formativo.

Penetrando, por tanto, en la niebla instalada, a la que con gran acierto se refieren Long y Siemens (2011), al afirmar sin fisuras que el análisis de los datos extraídos de la acción docente (LA) va a tener un papel significativo en el futuro de la Educación Superior, cabe señalar la incidencia tanto para: i) los profesores/educadores, que puedan conocer a tiempo real el comportamiento y rendimiento de los estudiantes e identificar a quienes muestren ritmos de aprendizaje más lentos, quienes estén en riesgo de abandonar el curso y/o necesiten ayuda para avanzar en el aprendizaje; ii) los estudiantes, por cuanto pueden recibir información particularizada de su progreso, siendo un elemento que les motive e incentive a seguir mejorando; y, iii) para los gestores o responsables, a quienes permite conocer la eficiencia de los recursos empleados, la existencia de nichos de ventajas competitivas, mejoras en la calidad y rendimientos de la experiencia de aprendizaje. Buckingham y Ferguson (2012) fijan como raíces del LA en dos hitos como son el desarrollo de la inteligencia de negocios sobre productos y servicios de e-learning y la minería de datos (también llamada Knowledge Discovery in Databases, KDD) y que se extiende a áreas de investigación en computación, aprendizaje bayesiano, redes neuronales artificiales, árboles de decisión, programación lógica, inducción de reglas, algoritmos estadísticos, etc. (Romero y Ventura, 2007). 
La extraordinaria proliferación de investigación de la última década, agrupada en comunidades de aprendizaje distintas, Minería de datos educativos (EDM) y Learning Analytics (LA) and Knowledge (LAK), ha llevado a Siemens y Baker (2012) a abogar por una mayor y formal comunicación y colaboración entre ellas, que si bien muestran diferencias en los descubrimientos, la adaptación y personalización, son perfectamente salvables y llevan a compartir investigaciones, técnicas, métodos y herramientas en la extracción y análisis de los datos, habiendo de facto entre ellas más intersección que espacios diferenciados. Stewart (2017) apunta que las investigaciones de LA abordan actividades en campos concretos de acción como la minería de datos, la inteligencia artificial, la analítica de registros web, así como la analítica de la inteligencia comercial, e implican la integración de datos de múltiples fuentes para informar sobre el uso de sistemas de gestión educativa como el Learning Management System (LMS), al que se refieren acertadamente Leah y Dawson (2010).

Adentrados en éste área de investigación, Romero y Ventura (2007) constituyen una guía y referencia imprescindible ${ }^{6}$ para afrontar la aplicación de la minería de datos en sistemas de e-learning en su ciclo iterativo, por cuanto ingresa al sistema educativo y guía, facilita y mejora el aprendizaje, tanto de forma genérica, generando conocimiento para los docentes y los propios estudiantes, como de forma particular para la toma de decisiones. Ferguson (2012) incide en los factores que explican el crecimiento exponencial del learning analytics en la última década, poniendo el foco en tres retos: la generación automática de grandes matrices de datos (big data), cuyo desafío técnico ya está superado por las metodologías de tratamiento de datos para extraer valor sobre el proceso del aprendizaje; el reto de optimizar las oportunidades del aprendizaje masivo en línea (online learning), y los intereses o inquietudes políticos (political concerns) y económicos por ofrecer mejores indicadores y resultados de aprendizaje, a nivel nacional e internacional. Dichos retos apuntan a tres colectivos cuyos intereses se superponen en diferentes escalas y con distintos grados de profundidad, profesores, instituciones educativas y

${ }^{6}$ A partir de numerosas referencias que aportan, sistematizan y clasifican en un catálogo estandarizado por tipo de datos, técnicas utilizadas y aspectos educativos que abordan, como son: Educación tradicional; Educación basada en la web y e-learning; Sistemas de gestión de aprendizaje; Sistemas Inteligentes de Tutoría; Sistemas Educativos Adaptativos; Pruebas y Cuestionarios; Textos y Contenido, y otros, y establecen una clasificación de los usos de la minería de datos en la analítica educativa: Análisis y visualización de datos; Predicción del rendimiento del alumno; Proporcionar retroalimentación para los instructores; Agrupación de estudiantes; Detectar conductas estudiantiles atípicas; Recomendaciones para estudiantes; Análisis de redes sociales; Modelado de estudiantes; Planificación y programación; Desarrollo de mapas conceptuales; Construcción de cursos. 
gobiernos, aspecto que se traduce en la importancia de elegir los problemas que los investigadores abordan e investigan y su traslación al público objetivo y sus intereses más directos.

Recogiendo la estela de autores previos, sin dejar de ser una referencia relevante, Sampson (2017) particulariza y clasifica los usos de los registros en líneas de investigación para i) definir perfiles, a partir de datos estáticos (aspectos demográficos, sociales, familiares, académicos) y/o datos dinámicos (referidas al proceso de aprendizaje como son el compromiso con el proceso, comportamiento, y rendimiento); y, ii) aplicar analítica del aprendizaje (LA), según un catálogo de métodos y herramientas base de los análisis descriptivos, predictivos y/o prescriptivos de recomendaciones.

Autores como Ferguson y Clow (2017) o Banihashem y otros (2018) se refieren a que desde la primera conferencia de LAK de 2011, la investigación parece centrarse principalmente en la medición, recopilación, análisis e informe de datos sobre los estudiantes, sus contextos e interactuaciones con los sistemas o herramientas, para justificar la mejor comprensión y la optimización del aprendizaje, modelar y generar perfiles de alumnos, así como formular un aprendizaje personalizado y adaptable (Stewart, 2017).

Son numerosos los autores que visualizan un futuro aprendizaje personalizado, sustentado en los beneficios de disponer evidencia empírica y parametrizar la información/datos de los estudiantes, referidos a su comportamiento, rendimiento y compromiso. Así, algunos autores evalúan la validez de herramientas o algoritmos de clasificación en la minería de datos ${ }^{7}$ para identificar a los estudiantes que tienen, por ejemplo, un ritmo más lento de aprendizaje, lo que proporciona una base adicional para diseñar programas específicos de ayuda temprana (Kaura, Singhb y Josanc, 2015).

Percibir el alcance de la fructífera investigación en Learning Analytics Ileva a revisar las múltiples implementaciones que ya son una realidad en diversos Centros Educativos y Universidades, tanto a través de registros de planes formativos formales (reglados) o informales, como muestran Ferguson, Brasher, Clow y otros (2016), quienes relacionan un catálogo exhaustivo de investigaciones analíticas a partir de casos de adaptación de tecnologías y juegos digitales de aprendizaje, así como buenas prácticas a imitar. Estas aplicaciones y posteriores analíticas tienen enfoques multidisciplinares, y se diseñan desde disciplinas con áreas de intersección como la estadística, la inteligencia artificial, la ciencia computacional, o desde el diseño de perfiles psicológicos y de aprendizaje, según apuntan Siemens, Dawson y Linch (2013).

${ }^{7}$ Como el Multi Layer Perception (MLP). 


\section{Metodología del estudio de caso}

Es frecuente encontrar estudios de caso de LA o empíricos con datos que provienen de las interacciones de los estudiantes con los sistemas o herramientas digitales, generados durante el tiempo de participación o uso, incidiendo en la importancia del tamaño y variedad de los datos para extraer información sobre el proceso de aprendizaje, con tintes de generalización y extrapolación, descripción de constructos explicativos más complejos e identificación de eventos o comportamientos anómalos (Banihashem y otros, 2018). Siguiendo algunos de los elementos previamente expuestos, en este apartado se describe el estudio de caso que corresponde al primer cuatrimestre del curso 2018-198 (de septiembre a enero) en la asignatura de Análisis Microeconómico Avanzado (AMA), de $4^{\circ}$ curso del Grado en Economía (GECO), en el itinerario de Análisis Económico (AE), y se dispone de un total de 25588 registros de uso digital, para 69 estudiantes inscritos. La investigación empírica se realiza a partir de diversas fuentes primarias de información recopilada, como son la información institucional del curso, los registros de usos en las plataformas digitales, controles de asistencia y calificaciones de evaluación continua y final. Se conjuga por tanto bases de datos de gran tamaño de actuaciones y tareas de aprendizaje, con datos que caracterizan a los individuos, ya que toda la información sobre los procesos de aprendizaje puede y debe ser considerada de manera integral (Lodge y Corrin, 2017).

\subsection{Registros de datos}

En la línea de lo expuesto por Sampson (2017), se han recuperado diversos instrumentos de información primaria, que aportan información complementaria:

- Los registros de los usos de las herramientas del campus virtual Moodle y del blog ${ }^{9}$ de la asignatura (en abierto y gestionado por el profesor), para lo cual se ha seguido el trabajo empírico de Vela-Pérez y otros (2017) como guía para la extracción, la descripción y el análisis de los datos, al estar referido a la misma plataforma Moodle.

- Las calificaciones según la reseña de evaluación explicitada en la guía docente, a partir de tres elementos diferenciados: 1) la

${ }^{8}$ En el curso 2017-18 se realizó un análisis parcial de los registros, a modo de prueba, con el único objetivo de aprender sobre los mismos, y planificar con antelación los elementos a trabajar para que la actual investigación, referida al curso 2018-19, no fuera fallida por falta de previsión.

${ }^{9}$ Blog o campus virtual en abierto. 
evaluación continua (EV) con un peso del $10 \%$ de la calificación final, correspondientes a seminarios grupales en el aula y su continuación como práctica, individual o grupal, y que se despliega en una estructura gamificada con asignación de tres insignias según ranking de desempeños en los seminarios grupales; 2) las pruebas de evaluación parcial (PEP) con un peso del $40 \%$ en conjunto, consistente en dos pruebas correspondientes a los bloques temáticos del curso, y 3) la prueba de evaluación final (PEF) del curso, que completa el 50\% restante de la calificación del estudiante.

Así, el cuadro 1 muestra en detalle el cronograma de actividades, pruebas y gamificación, en las 15 semanas efectivas (con carga docente presencial) del curso, que se corresponden con el patrón de uso de los estudiantes del aula virtual.

Cuadro 1. Cronograma según actividades de evaluación

\begin{tabular}{|c|c|c|c|c|}
\hline \multirow{2}{*}{$\frac{\text { Semana }}{1}$} & $\begin{array}{c}\text { Seminarios (SEM) } \\
\text { Grupales } \\
\text { Evaluación } \\
\text { continua (EVC) }\end{array}$ & $\begin{array}{l}\text { Gamificacion: } \\
\text { Insignias, } \\
\text { privilegios }\end{array}$ & $\begin{array}{c}\text { Pruebas } \\
\text { parciales } \\
\text { (PEP) }\end{array}$ & \multirow[t]{2}{*}{$\begin{array}{l}\text { Prueba final } \\
\text { (PEF) }\end{array}$} \\
\hline & & & & \\
\hline 2 & SEM 1+ PRÁCTICA & & & \\
\hline 3 & & & & \\
\hline 4 & SEM 2 + PRÁCTICA & & & \\
\hline 5 & & & & \\
\hline 6 & SEM 3 + PRACTICA & & & \\
\hline 7 & & & & \\
\hline 8 & SEM 4 + PRÁCTICA & INSIGNIA AMARILLA & & \\
\hline 9 & REPASO & & & \\
\hline 10 & & & PEP 1 & \\
\hline 11 & & & & \\
\hline 12 & SEM 5 + PRÁCTICA & INSIGNIA ROJA & & \\
\hline 13 & & & & \\
\hline 14 & & & PEP 2 & \\
\hline 15 & SEM 6 + PRÁCTICA & INSIGNIA VERDE & & PREPARACIÓN \\
\hline
\end{tabular}

Es necesario explicitar que i) los estudiantes han sido informados y tienen la oportunidad de solicitar que sus registros no sean incluidos en el análisis, son conocedores de la naturaleza de los datos que la plataforma registra, y por tanto consienten o no el seguimiento de su huella o rastro en el aprendizaje, así como el cruce con informaciones de otras fuentes, como son los datos institucionales de identificados y convocatoria, ii) se garantiza que a los estudiantes al consentir la observación de sus registros no les suponga ningún compromiso, obligación, disponibilidad y dedicación adicional, y iii) 
la extracción y cruce de la información se realiza bajo un algoritmo estadístico que, tras la recodificación de los ítems de identificación personal, automáticamente hace anónima la información del estudiante ${ }^{10}$, cumpliendo con la normativa vigente de protección de datos de los usuarios o estudiantes.

Cabe reconocer que la existencia de poderosas herramientas de gestión de datos, con un alcance cada vez mayor, está planteado entre los diversos colectivos (usuarios y responsables) la preocupación sobre aspectos muy pertinentes referidos a cuestiones éticas, de privacidad y protección, abordados por ejemplo en Campbell ( $\left.2007^{11}\right)$, aspectos que se respetan en ésta investigación dado que toda la información ha sido anonimizada previamente.

Respecto a las herramientas, si bien en la literatura empírica de última década se referencian una gran cantidad de herramientas, tanto generales como específicas de minería de datos, que van desde programas comerciales estandarizados (DBMiner, SPSS DB2 Intelligent Miner), a numerosas aplicaciones de uso público desarrolladas por colectivos de investigadores (algunas disponibles en abierto y de uso libre (por ejemplo GISMO, Google analytics, Smartklass), en ésta primera explotación la gestión de los datos se realiza con herramientas básicas del paquete estadístico SPSS, siendo una referencia el trabajo de Romero, Ventura y García (2008).

\subsection{Población de análisis y ficha técnica}

Describir brevemente las características de la población objeto del estudio contextualiza el análisis posterior, para lo cual a modo de ficha técnica en la tabla 1 se aportan varios indicadores como la nacionalidad y el género del estudiante, junto con la convocatoria en curso y el porcentaje registrado de asistencia en la asignatura de referencia.

Así, según la identificación oficial, el $65,22 \%$ del total de estudiantes tienen DNI de nacionalidad española, y el resto, 34,78\%, extranjera, por cuanto que su documentación es NIE, entre los que cabe distinguir dos tipologías: aquellos identificados con doble nacionalidad (por segunda generación, o con tarjeta de residencia), y los que tienen nacionalidad China, que representan el $24,63 \%$ del total de estudiantes y el $78,83 \%$ de los identificados con NIE de extranjero.

10 Conciliar la investigación y las normas europeas de protección de datos (Europe's General Data Protection Regulation, GDPR, de 2016) es necesario y está generando cambios en el uso futuro del Learning Analytics, por cuanto las instituciones tienen responsabilidad y obligaciones en dicha protección.

${ }^{11}$ Examina más de 70.000 registros para determinar los indicadores más sólidos (factores robustos) del éxito de los estudiantes, a partir de modelos de regresión estadística. 
Tabla 1. Distribución de estudiantes AMA según información institucional.

\begin{tabular}{|c|c|c|c|c|}
\hline Identificador & Nacionalidad & $\begin{array}{c}\text { Género Varones } \\
\text { (\%) }\end{array}$ & $\begin{array}{c}1^{a} \\
\text { Convocatoria }\end{array}$ & $\begin{array}{c}\text { Asistencia } \\
(\%)\end{array}$ \\
\hline \multirow[t]{2}{*}{ DNI español } & $N_{1}=45(65,22 \% N)$ & $31(44,93 \%$ de $N)$ & $35(50,72 \% N)$ & $71,5 \%$ \\
\hline & & {$\left[75,61 \%\right.$ de $\left.N_{1}\right]$} & $\left(77,77 \%\right.$ de $\left.N_{1}\right)$ & \\
\hline \multirow[t]{2}{*}{ NIE extranjero* } & $\mathrm{N}_{2}=24(34,78 \% \mathrm{~N})$ & $10(14,493 \%$ de $N)$ & $20(28,99 \% N)$ & $64,9 \%$ \\
\hline & & {$\left[41,67 \%\right.$ de $\left.\mathrm{N}_{2}\right]$} & $\left(83,33 \%\right.$ de $\left.N_{2}\right)$ & $\begin{array}{l}(83,84 \% \text { no } \\
\text { chino) }\end{array}$ \\
\hline \multirow[t]{2}{*}{ NIE origen China } & $\mathrm{N}_{21}=17 \quad(24,63 \%$ & $5(7,55 \% N)$ & $13(18,84 \% \mathrm{~N})$ & $57,1 \%$ \\
\hline & & {$\left[29,41 \%\right.$ de $\left.N_{21}\right]$} & $\left(76,40 \% \mathrm{~N}_{21}\right)$ & \\
\hline \multirow[t]{2}{*}{ Total } & $\mathrm{N}=69$ & $41(59,42 \% N)$ & $55(79,71 \% N)$ & $69,2 \%$ \\
\hline & & 28 mujeres & & \\
\hline
\end{tabular}

* Según letra del identificador, DNI, de los estudiantes que no son de nacionalidad china, 3 se corresponden con estudiantes Erasmus in, y el resto son estudiantes con doble nacionalidad o residentes.

El 59,42\% de los estudiantes del curso AMA son varones, lo cual muestra una ligera diferencia en la selección de la especialidad de Análisis, respecto del $64,0 \%$ de varones de la titulación. Segmentando, el 44,93\% del total de estudiantes tienen DNI de nacionalidad española y son varones (el 75,61\% de los que presentan DNI nacional son varones), mientras que los que tienen NIE y varones son el $14,61 \%$ (el $41,67 \%$ de los que tienen NIE son varones, mientras que entre los estudiantes de nacionalidad china solo el 29,41\% son varones). Por lo que respecta a la convocatoria que cursan, el 79,71\% están inscritos en la primera, y por segmentaciones, el 50,72\% tienen DNI y están en la primera convocatoria, mientras que el $28,99 \%$ tienen NIE y están en la primera convocatoria. EI $77,77 \%$ de los que tienen DNI están en la primera convocatoria, y el $83,33 \%$ de los que tienen NIE, y por género se distribuyen de forma que el $61,81 \%$ de los que están en primera convocatoria son varones frente al 38,18\% que son mujeres, y complementariamente, el 82,93\% de los varones están en la primera convocatoria y el $75 \%$ de las mujeres.

En cuanto al porcentaje de asistencia a las clases presenciales, la asistencia media en más de 14 registros semanales aporta una media de $69,2 \%$, con diferencial según nacionalidad, por cuanto quienes tienen DNI asisten en media el 71,5\%, y los que tienen NIE asisten el 64,9\%, si bien con comportamientos polarizados, con un $83,8 \%$ los que no tienen nacionalidad China (incluyéndose a los estudiantes Erasmus) y 57,1\% los que sí lo tienen, siendo estos estudiantes los más absentistas ${ }^{12}$. Estos resultados no están completamente alineados con los datos institucionales (Facultad de CCEE, UCM), según los cuales identifican con el 65,2\% de participación media en $4^{\circ}$ grado de ECO, y un $55,5 \%$ en el área de estudio.

12 El problema del absentismo entre la comunidad de estudiantes chinos se hace crónica con las limitaciones del uso del idioma, que siguen programas de estudio no reglados con preparadores de origen chino. 
Según el cronograma semanal, en la semana 10 del curso (transcurrido el 66,6\% del mismo), el 8,7\% de los estudiantes (6) había cuasiabandonado la evaluación continua en el aula, uno de ellos la segunda semana del curso. En el desarrollo metodológico del curso, los estudiantes participan en seminarios grupales de trabajo (3 o 4 estudiantes), que en general se autoseleccionan, por conocimiento y empatía, sólo en algunas ocasiones lo son por la observación previa de su complementariedad competencial, siendo relevante la supervisión que el docente hace del proceso de adscripción en grupos, por cuanto puede hacer que el aprendizaje sea o no más motivador y cognitivamente enriquecedor (Calzadilla, 2002), evitando que existan polarizaciones grupales que estrangulen el rendimiento medio, así como favorecer una mayor integración entre los colectivos por nacionalidad.

Cabe señalar dos aspectos adicionales: 1) No se dispone información directa sobre la edad de los estudiantes, pero la convocatoria en la que están es una aproximación de los años que Ilevan en la Universidad, dado que al ser una asignatura de $4^{\circ}$ curso, los estudiantes en primera convocatoria (casi sin error) están en el cuarto año de permanencia; 2) No es significativo ${ }^{13}$ realizar un análisis de diferencias entre los diversos subgrupos por edad, nacionalidad, experiencia y género, dada la reducida adscripción de estudiantes a los mismos, si bien la percepción subjetiva revela un grado de madurez personal, experiencial y laboral diverso.

\section{Resultados del seguimiento y registro de usos}

Se presentan algunos de los resultados de la asignatura AMA en el curso 2018-19, tanto desde la descripción del rastreo o huella que los estudiantes dejan en su uso activo del campus virtual y el blog, como la interacción con las calificaciones de los diversos elementos evaluables.

\subsection{Registro de usos en las plataformas virtuales por estudiante.}

El total de estudiantes, tabla 2, contabiliza 25588 registros válidos (recuperados a posteriori, finalizado el periodo lectivo del curso, el 22 de diciembre de 2018) de las platafomas digitales activas en el curso. Como indica Siemens (2013), el uso de dispositivos móviles, herramientas virtuales y medios sociales en el proceso de aprendizaje genera huellas en senderos

${ }^{13}$ El test de diferencias no es significativo dada la limitación de las submuestras. 
digitales, incluyendo patrones de navegación, pausas, hábitos de lectura y hábitos de escritura, que para el docente/investigador ofrecen una oportunidad para observar el aprendizaje desde múltiples ángulos.

Una visión sintética del porcentaje de registros por estudiantes en media semanal respecto del total acumulado, muestra la elevada dispersión, en los usos de las plataformas digitales (véase gráfico 1 y 2). Los 10 estudiantes más activos registran en media porcentajes entre el 4,19 y el 2,61\% del total, con accesos semanales respectivos de 71,53 a 44,60, mientras que los 10 menos activos están entre el 0,51 y el 0,00\% del total, con accesos semanales de 8,67 a 0 , siendo la media del total de estudiantes de 24,37 accesos semanales a las distintas páginas, representando el 1,43\% del total de registros, con una desviación en promedio de 180,73.

Tabla 2. Estadísticos total registros y brecha de uso semanal

\begin{tabular}{|c|c|c|c|c|c|c|}
\hline $\begin{array}{l}10 \text { más } \\
\text { activos }\end{array}$ & $\mathrm{N}$ registros & $\%$ del total & $\begin{array}{c}\text { I } \\
\text { Media } \\
\text { semanal }\end{array}$ & $\begin{array}{c}\text { II } \\
\text { Dife- } \\
\text { rencial } \\
\text { respecto } \\
\text { media \% }\end{array}$ & $\begin{array}{c}\text { III } \\
\text { Brecha } \\
\text { respecto de } \\
\text { la media \% }\end{array}$ & $\begin{array}{c}\text { IV } \\
\text { Brecha } \\
\text { respecto el } \\
\text { A12 }\end{array}$ \\
\hline A12 & 1073 & 4,19 & 71,53 & 47,16 & 193,50 & 0,00 \\
\hline A51 & 1018 & 3,98 & 67,87 & 43,5 & 178,46 & 5,13 \\
\hline A69 & 976 & 3,81 & 65,07 & 40,7 & 166,97 & 9,04 \\
\hline A8 & 755 & 2,95 & 50,33 & 25,96 & 106,52 & 29,64 \\
\hline $\mathrm{A} 2$ & 714 & 2,79 & 47,60 & 23,23 & 95,30 & 33,46 \\
\hline A17 & 713 & 2,79 & 47,53 & 23,16 & 95,03 & 33,55 \\
\hline A20 & 694 & 2,71 & 46,27 & 21,9 & 89,83 & 35,32 \\
\hline A55 & 681 & 2,66 & 45,40 & 21,03 & 86,28 & 36,53 \\
\hline A16 & 679 & 2,65 & 45,27 & 20,90 & 85,73 & 36,72 \\
\hline A29 & 669 & 2,61 & 44,60 & 20,23 & 82,99 & 37,65 \\
\hline $\begin{array}{l}10 \text { menos } \\
\text { activos }\end{array}$ & registros & $\%$ del total & $\begin{array}{l}\text { Media } \\
\text { semanal }\end{array}$ & $\begin{array}{c}\text { Dife- } \\
\text { rencial } \\
\text { respecto } \\
\text { media \% }\end{array}$ & $\begin{array}{c}\text { Brecha } \\
\text { respecto de } \\
\text { la media \% }\end{array}$ & $\begin{array}{c}\text { Brecha uso } \\
\text { semanal }\end{array}$ \\
\hline A33 & 130 & 0,51 & 8,67 & $-15,7$ & $-64,44$ & 87,88 \\
\hline A59 & 76 & 0,30 & 5,07 & $-19,3$ & $-79,21$ & 92,92 \\
\hline A44 & 76 & 0,30 & 5,07 & $-19,3$ & $-79,21$ & 92,92 \\
\hline A41 & 68 & 0,27 & 4,53 & $-19,84$ & $-81,40$ & 93,66 \\
\hline A62 & 51 & 0,20 & 3,40 & $-20,97$ & $-86,05$ & 95,25 \\
\hline A7 & 36 & 0,14 & 2,40 & $-21,97$ & $-90,15$ & 96,64 \\
\hline A24 & 34 & 0,13 & 2,27 & $-22,1$ & $-90,70$ & 96,83 \\
\hline A61 & 9 & 0,04 & 0,60 & $-23,77$ & $-97,54$ & 99,16 \\
\hline $\mathrm{AO}$ & 4 & 0,02 & 0,27 & $-24,1$ & $-98,91$ & 99,63 \\
\hline A54 & 0 & 0,00 & 0,00 & $-24,37$ & $-100,00$ & 100,00 \\
\hline Todos, Media & 25588 & 1,43 & 24,37 & - & - & 65,63 \\
\hline
\end{tabular}




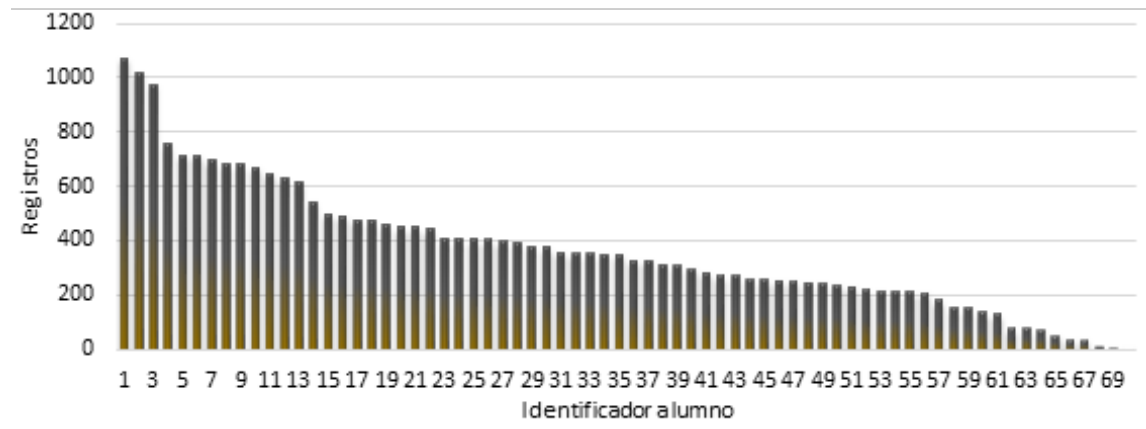

Gráfico 1. Registros ordenados de mayor a menor, total de estudiantes

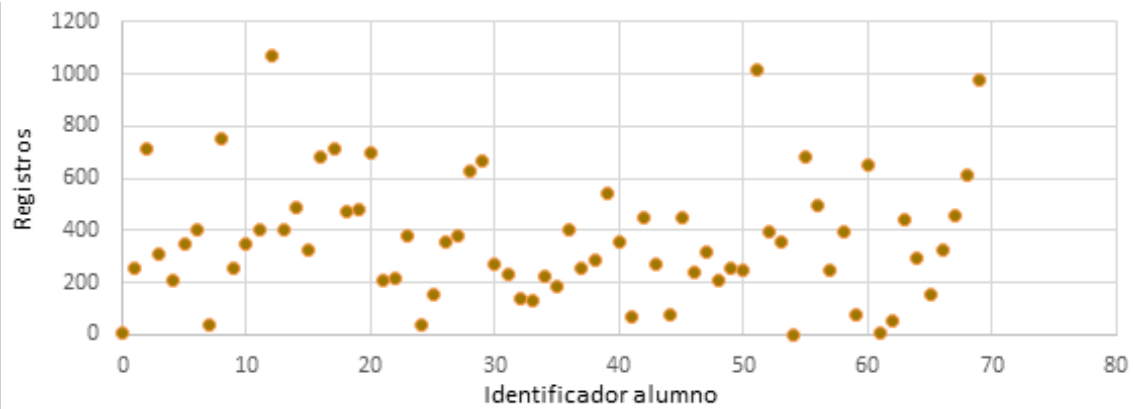

Gráfico 2. Dispersión de registros por alumno

Como puede observarse, los alumnos exhiben muy diversa frecuencia, presencia y actividad en las diversas herramientas electrónicas que se disponen, cuyo ritmo difiere por semanas e incluso por días, según la actividad docente programada. Estos diferenciales de uso determinan el cálculo del indicador de "brecha de uso digital", que facilita una visión más completa del patrón de uso el curso (total de registros en el Anexo) a partir de una clasificación y tipología básica de los estudiantes en varias categorías y que se muestra en los gráficos 3 y 4 adjuntos. Así, si el indicador se calcula referenciado al estudiante más activo, el $\mathrm{A} 12$, (columna $\mathrm{V}$ tabla 1 ), lógicamente hace que el peso de la mayoría de los estudiantes caiga hacia el extremo de poca o escasa actividad registrada dando una visión con fuerte asimetría:

a) proactivos: si registran una brecha $<20 \%$, en la que sólo entran 3 alumnos: A12, A51 y A69, y con media de 4,721.

b) sobreactivos: brecha entre 20 y $40 \%$, que incluye a 8 alumnos, y con media de 35,31.

c) medio activos: una brecha entre 40 y el $60 \%$, a la que se adscriben sólo 11 alumnos con media de 53,30.

d) subactivos: con brecha entre 60 y $80 \%$, que incluye a 30 alumnos con una media de 70,45.

e) inactivos o descolgados: si la brecha está entre el 80 y 100\%, que agrupa a 18 alumnos cuya media es 89,91. 
Por el contrario, si el cálculo del indicador de la brecha digital se realiza sobre el valor medio de la distribución (columna IV tabla 1), el alumno más centrado sería el A27 (brecha de 2,58), y la clasificación obtenida aporta una visión más dulce de los usos registrados:

a) proactivos: si registran una brecha positiva de $>75 \%$, en la que se incluyen 11 alumnos, con media de 118,06.

b) sobreactivos, con una brecha positiva entre 75 a $25 \%$, en la que se incluyen 7 alumnos, con media de 49,24.

c) medio activos, con una brecha entre +25 y $-25 \%$, en la que se incluyen 23 alumnos, que registran media de 1.91

d) subactivos, con brechas negativas entre el 25 y $75 \%$, que aglutina a $2^{\circ}$ estudiantes.

e) inactivos o descolgados, con brechas negativas superiores al 75\%, con 9 estudiantes.

Dado este perfil de usos, sea cual sea el cálculo del indicador hecho para identificar la brecha digital, como criterio de mejora para cursos posteriores se deben supervisar los registros de forma casi automática en períodos diversos, que permita un seguimiento continuado para recuperar a ritmos óptimos a aquellos estudiantes que evidencian signos de subactividad o descolgamiento del curso, especialmente tras la primera prueba de evaluación como medida preventiva.

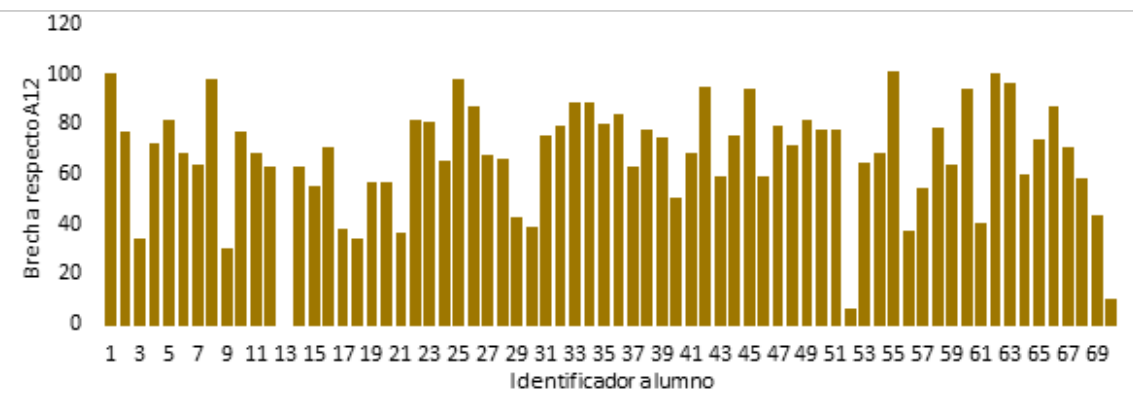

Gráfico 3. Brecha porcentual registros respecto del alumno más activo A12 (en positivo)

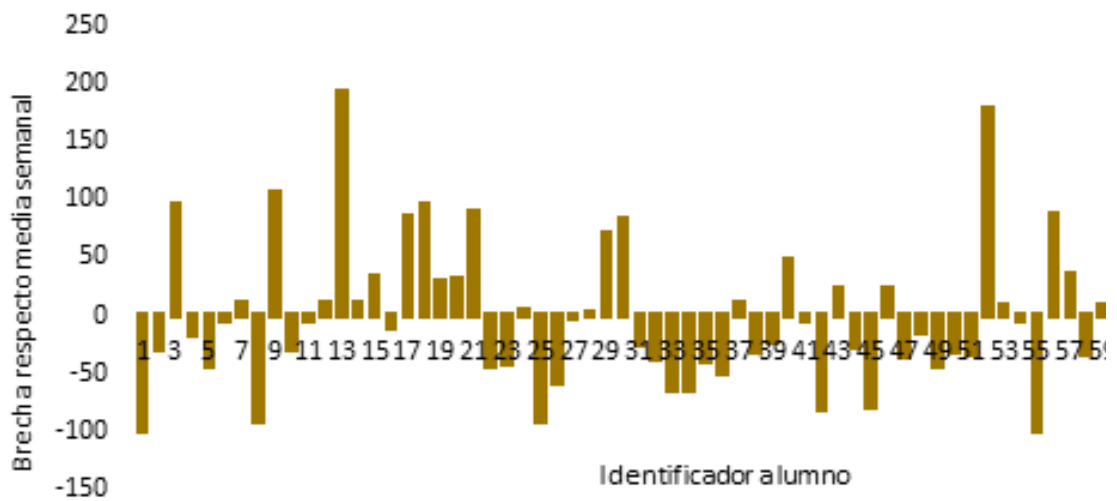

Gráfico 4. Brecha porcentual registros respecto de la media semanal 


\subsection{Temporalidad de la distribución de registros}

En cuanto a la temporalidad de los accesos, según se observa en la tabla 3, existen diferencias notables en la distribución semanal, con una media ajustada de 1265 registros semanal por alumno, con picos de actividad del $17,16 \%$ del total en la semana 10 y del $12,75 \%$ en la semana 14 , que se corresponden con las pruebas parciales de evaluación (si bien los accesos se concentran en un $65 \%$ los días antes y el resto los días posteriores, para la comprobación de soluciones y calificaciones). En el arco opuesto, es muy reducida la actividad en torno al $1,40 \%$ en las semanas 9 y 18 , registrándose la mínima actividad, con el 0,77\% del total en la semana 17, de tránsito, exhibiendo una desviación respecto del promedio muy alta, de 14,60, y superior al promedio de registros por estudiante y semana, en las semanas 1, 2, 5, 9 y 18. De forma acumulativa, las cuatro primeras semanas (de 1 a 4) computan el $20,31 \%$ del total de registros, las siguientes 4 (de 5 a 8 ) el $12,79 \%$ y las semanas 9 a 12 agrupan el 27,19\% de los registros.

Tabla 3. Total registros por semanas y tipología, en porcentaje

\begin{tabular}{lccccccccc}
\hline & R_1 & R_2 & R_3 & R_4 & R_5 & R_6 & R_7 & $\begin{array}{c}\text { Media } \\
\text { registros } \\
\text { semanal }\end{array}$ & $\begin{array}{c}\text { Desviación } \\
\text { respecto } \\
\text { media }\end{array}$ \\
\hline Sem 1 & 54,18 & 0,00 & 0,56 & 2,23 & 42,48 & 0,00 & 0,00 & 10,30 & 11,79 \\
Sem 2 & 47,12 & 0,76 & 4,34 & 0,53 & 47,07 & 0,00 & 0,18 & 24,34 & 25,21 \\
Sem 3 & 50,35 & 2,19 & 0,35 & 0,15 & 45,92 & 0,50 & 0,55 & 28,74 & 23,24 \\
Sem 4 & 52,23 & 4,45 & 0,00 & 0,00 & 42,28 & 0,00 & 1,05 & 10,91 & 8,57 \\
Sem 5 & 53,32 & 0,77 & 0,00 & 1,15 & 44,64 & 0,00 & 0,13 & 5,46 & 7,00 \\
Sem 6 & 50,13 & 1,64 & 1,04 & 0,09 & 46,42 & 0,00 & 0,69 & 16,56 & 13,83 \\
Sem 7 & 53,94 & 1,27 & 0,00 & 0,00 & 43,77 & 0,00 & 0,76 & 5,6 & 4,57 \\
Sem 8 & 56,75 & 0,32 & 0,00 & 0,32 & 40,69 & 0,86 & 1,07 & 13,34 & 11,20 \\
Sem 9 & 51,79 & 3,58 & 0,28 & 2,75 & 41,60 & 0,00 & 0,00 & 5,19 & 9,58 \\
Sem 10 & 52,81 & 1,94 & 0,61 & 0,39 & 43,73 & 0,00 & 0,52 & 62,73 & 41,93 \\
Sem 11 & 48,35 & 6,58 & 0,62 & 0,62 & 43,72 & 0,00 & 0,10 & 13,89 & 12,41 \\
Sem 12 & 51,67 & 2,12 & 0,16 & 0,98 & 44,18 & 0,73 & 0,16 & 17,56 & 12,91 \\
Sem 13 & 53,34 & 1,81 & 0,57 & 0,79 & 42,58 & 0,79 & 0,11 & 12,61 & 9,88 \\
Sem 14 & 51,16 & 1,72 & 0,37 & 1,96 & 44,14 & 0,21 & 0,43 & 46,60 & 35,99 \\
Sem 15 & 51,50 & 2,81 & 0,19 & 1,44 & 43,94 & 0,00 & 0,13 & 22,80 & 16,34 \\
Sem 16 & 46,51 & 1,72 & 1,15 & 4,02 & 44,40 & 2,11 & 0,10 & 14,93 & 13,83 \\
Sem 17 & 51,53 & 0,51 & 0,00 & 0,00 & 44,39 & 0,00 & 3,57 & 2,80 & 2,69 \\
Sem 18 & 43,73 & 0,28 & 0,00 & 0,28 & 55,43 & 0,00 & 0,28 & 5,13 & 6,85 \\
Media por & 51,13 & 1,92 & 0,57 & 0,98 & 44,52 & 0,29 & 0,55 & 17,75 & 14,88 \\
registro & & & & & & & & &
\end{tabular}


Más aún, mientras que los días con docencia han sido lunes y martes, los días de la semana que registran un porcentaje mayor de accesos es el jueves y el domingo, con el $26,85 \%$ y $20,81 \%$ respectivamente, mientras que los días más inactivos son el sábado y el miércoles, con el $5,58 \%$ y el $6,28 \%$, temporalidad condicionado a la entrega de prácticas o ejercicios.

Es decir, los estudiantes conectados o no, realizan accesos a las plataformas virtuales a un ritmo condicionado, con ciertos desfases dentro de la misma semana, al cronograma de actividades planificadas durante el curso, y cuyo diferencial se observa en el gráfico 5 adjunto, con dispersiones muy altas entre sí, según tanto su tipología de uso como el avance temporal del curso. Reducir ésta dispersión puede ser un objetivo plausible para cursos venideros, acondicionando mejor los ritmos de los usos y sus registros a la dinámica de aprendizaje diseñada en el curso.

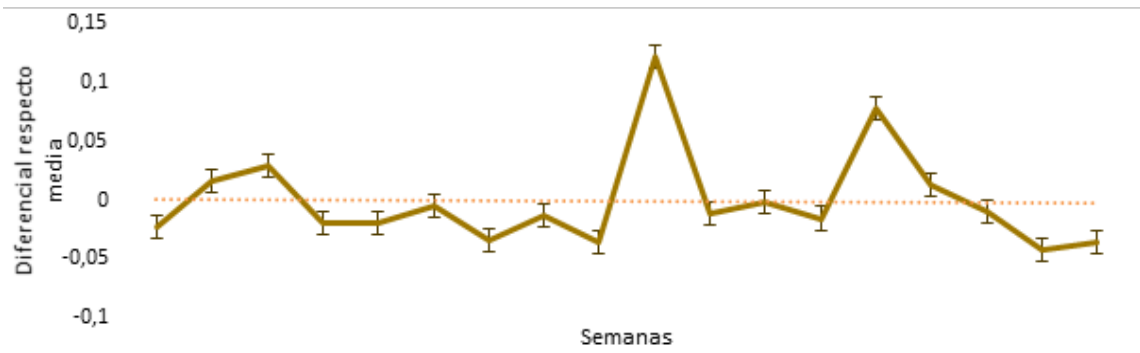

Gráfico 5. Perfil diferencial semanal respecto media

Los gráficos siguientes, abordan la distribución semanal de los registros, aspecto que refuerza la visión ya señalada de patrones diferenciados, por estudiante, semana y tipología de registro. Así, el gráfico 6 muestra el acumulado en los registros semanales de los estudiantes, cuyo perfil se corresponde, necesariamente con el del gráfico 2 .

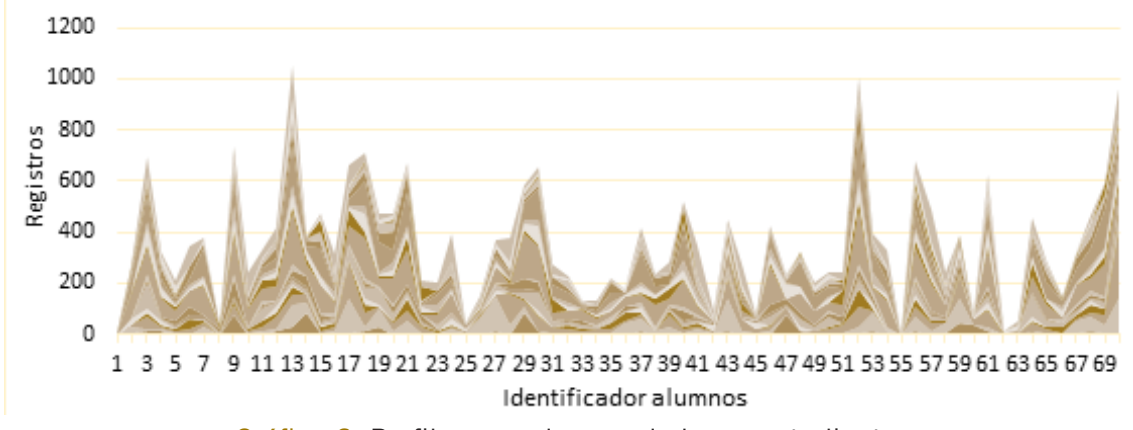

Gráfico 6. Perfil semanal acumulado por estudiantes 
Mirada aparte requiere la distribución de los estudiantes por semanas, y así el gráfico 7 muestra paralelamente las 4 primeras y últimas semanas del curso, por alumnos, con indicadores de dispersión. No son los mismos estudiantes y en las mismas semanas las que aportan valores extremos, como puede verse en la selección de semanas, quizás condicionado al tipo de trabajo grupal que se proyecta y que asigna ciertas responsabilidades de gestión intergrupal.
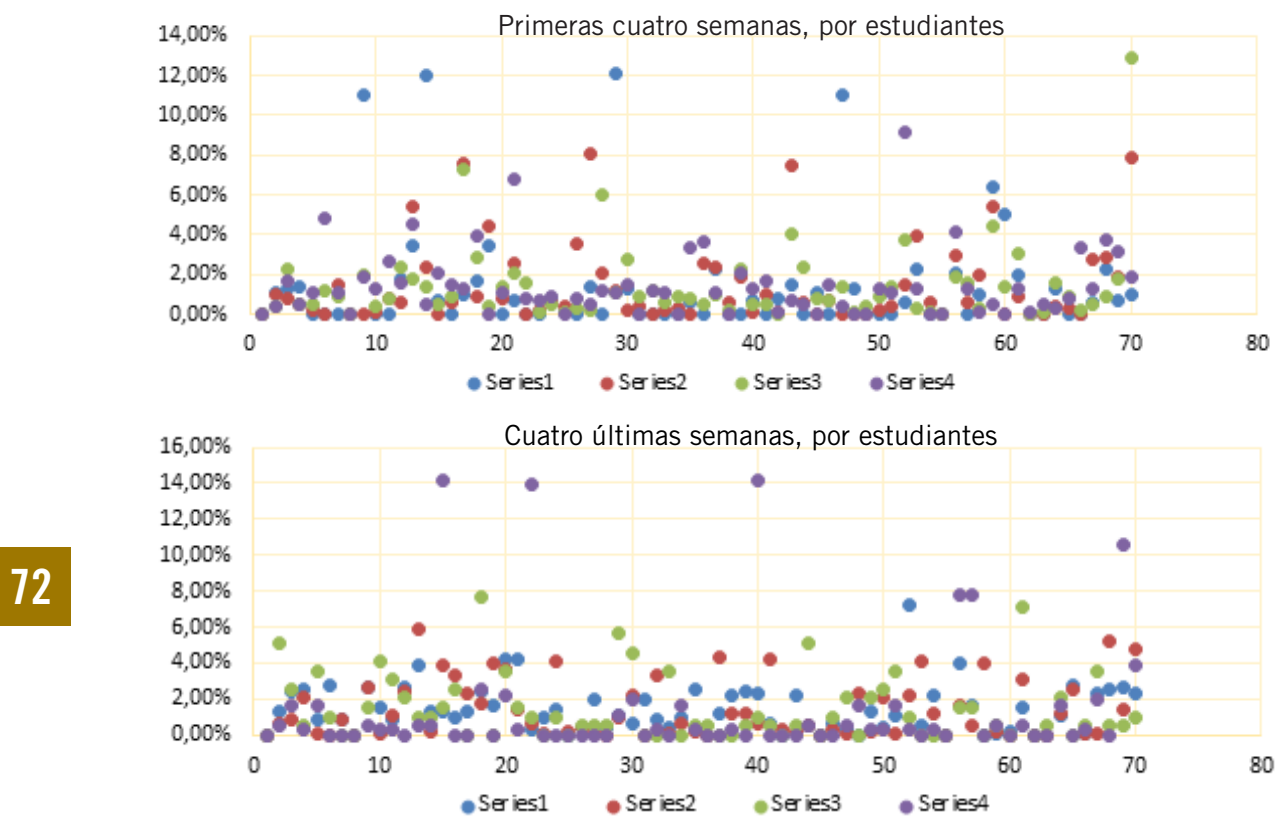

Gráfico 7. Dispersión registros semanal porcentual, por estudiantes.

Por lo que respecta a la naturaleza de los recursos, para su tabulación se han recodificado determinando la tipología que muestra la tabla 4. Así, para los estudiantes y en media, en torno a la mitad de los accesos que se realizan $(51,13 \%)$ son de carácter ordinario, a correos, información, noticias, nuevos temas o revisión, en torno al $44,5 \%$ de los accesos que registran los hacen tanto a los epígrafes de tareas evaluables como de recursos disponibles para su estudio y preparación, de forma que el resto del $4,37 \%$ de sus registros se concentran en las tareas evaluables $(1,92 \%)$, y el resto se reparte en porcentajes mínimos, siendo la participación más minoritaria en el foro interno del campus virtual, con sólo el 0,29\%. Esta información se muestra complementariamente en los gráficos 8,10 y 11 , y permite afirmar que el estudiante conectado sigue siendo tradicional en los usos que hace de las plataformas, polarizado en su actividad continuada, al estar básicamente pendiente tanto de consultar puntualmente la información y novedades que 
aporta el profesor en las plataformas virtuales y en el correo interno, como del acceso y la visualización de los materiales complementarios que le permiten estudiar y preparar las actividades evaluables en el curso.

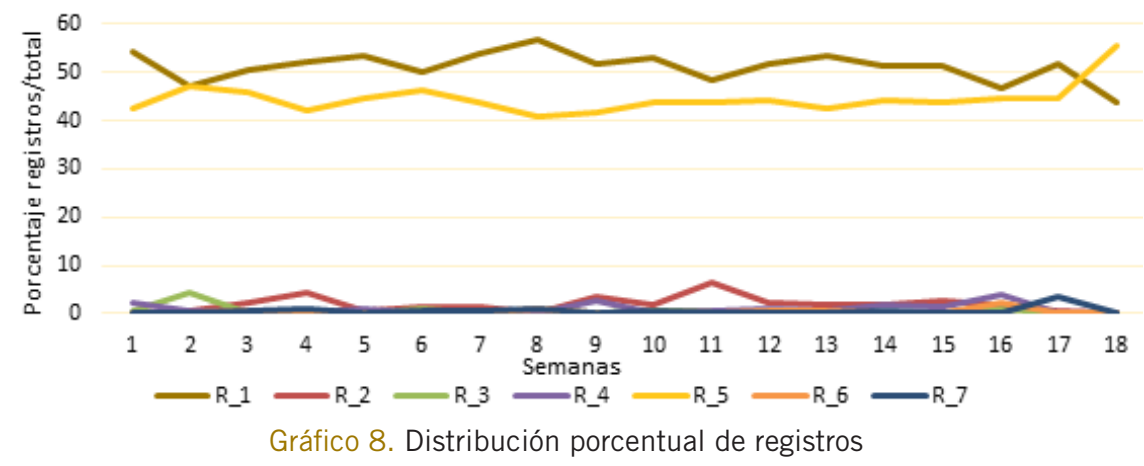

Esa visión estrangulada del uso polarizado del estudiante contrasta parcialmente si se compara con la distribución de la actividad registrada del profesor del curso, observándose un diferencial significativo en los usos no siempre acompasados por el alumno, que dibujan un perfil del profesor y estudiante muy diverso (gráfico 9 y tabla 4), lo que muestra que el profesor realiza un uso más diverso y radial de las diversas herramientas virtuales disponibles, tratando en muchos casos de dinamizar al grupo de estudiantes, si bien ellos no siempre siguen la estela.

Por tanto, el perfil de usos por alumno y profesor difiere tanto en el cómputo de registros como en la distribución de los mismos, lo cual se explica parcialmente por los roles internos que adquieren los estudiantes en las actividades grupales y el menor interés por determinadas actividades no evaluables.

Esta polaridad es causa, de la recodificación en la tipología de recursos extraídos, según naturaleza, que sin duda podría haberse hecho de otra forma, si bien su visión aportaría igualmente un uso tradicional de las herramientas, principalmente del campus virtual.

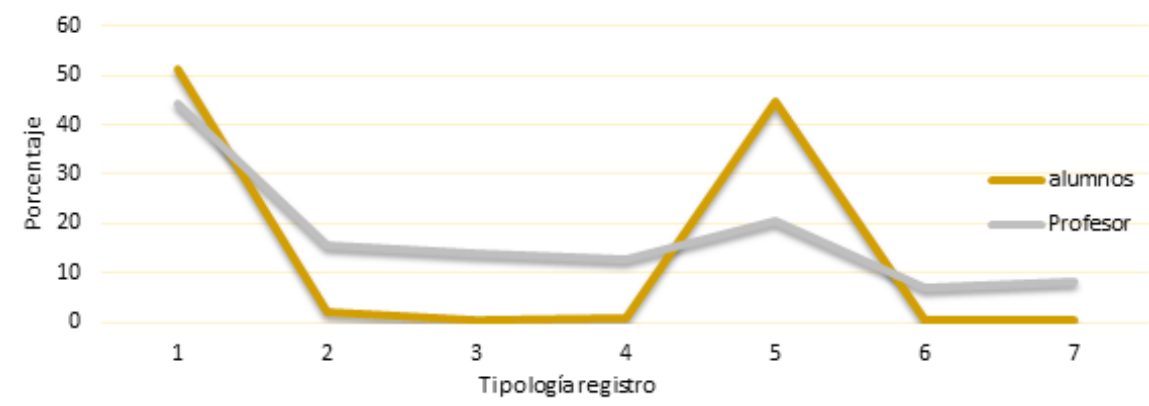

Gráfico 9. Distribución porcentual registros según tipología, por profesor y alumno. 
Tabla 4. Tipología y total de registros curso.

\begin{tabular}{|c|c|c|c|c|c|}
\hline \multirow[b]{2}{*}{$\begin{array}{c}\text { Tipología Registro } \\
\text { internet }\end{array}$} & \multicolumn{2}{|l|}{ Alumnos (A) } & \multicolumn{2}{|c|}{ Profesor (P) } & \multirow{2}{*}{$\begin{array}{l}\text { Diferencial } \\
\% \text { Alumnos } \\
\text { y profesor }\end{array}$} \\
\hline & $\begin{array}{l}\text { Media estudiante } \\
\text { /semana }\end{array}$ & $\%$ & $\begin{array}{l}\text { Media / } \\
\text { semana }\end{array}$ & $\%$ & \\
\hline General y sistema $=1: R_{-} 1$ & 9,55 & 51,13 & 29,75 & 44,40 & 6,73 \\
\hline Tareas evaluables=2: R_2 & 0,37 & 1,92 & 10,35 & 15,45 & $-13,93$ \\
\hline $\begin{array}{l}\text { Filiación e identificación=3: } \\
\text { R_3 }\end{array}$ & 0,14 & 0,57 & 9,3 & 13,88 & $-13,31$ \\
\hline Informes agrupados=4: R_4 & 0,17 & 0,98 & 8,6 & 12,84 & $-11,89$ \\
\hline Recursos disponibles=5: R_5 & 8,19 & 44,52 & 13,75 & 20,52 & 24,01 \\
\hline Foros y chats=6: R_6 & 0,05 & 0,29 & 4,65 & 6,94 & $-6,65$ \\
\hline $\begin{array}{l}\text { Archivos compartidos e Infor- } \\
\text { mes evaluadores =7: R_7 }\end{array}$ & 0,08 & 0,55 & 5,6 & 8,36 & $-7,81$ \\
\hline Total & 18,54 & 100 & 67 & 100 & - \\
\hline
\end{tabular}

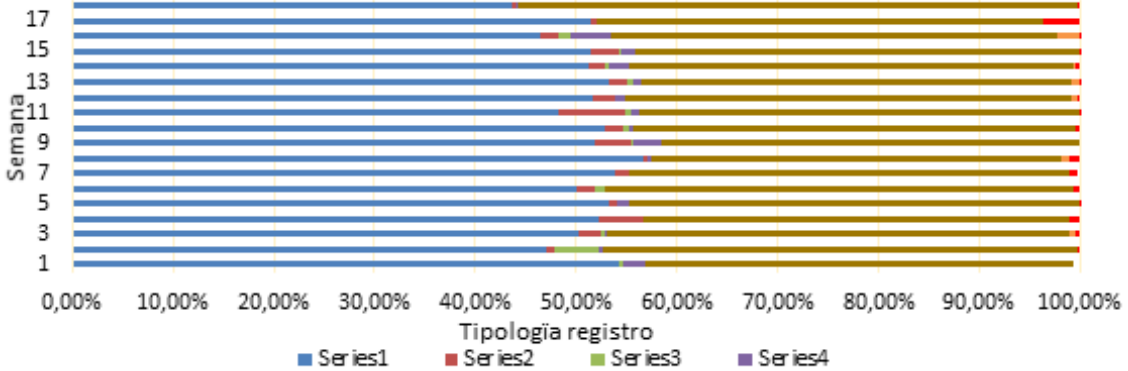

Gráfico 10. Distribución porcentual según tipología, por semana.

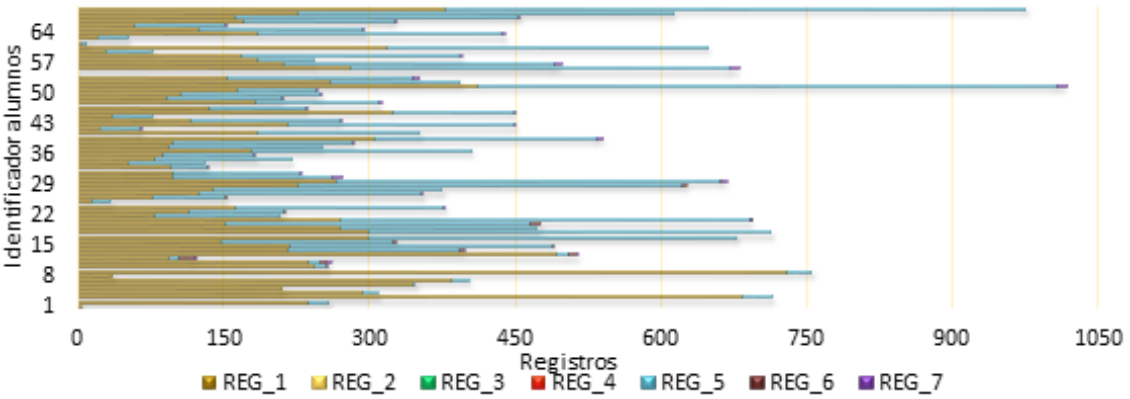

Gráfico 11. Distribución acumulada de registros por tipología y alumno. 


\subsection{Análisis de la evaluación continua y la calificación del curso AMA}

Como se ha indicado, los elementos que entran dentro de la calificación final de los estudiantes son: EVC; PEP y PEF, con pesos relativos del $10 \%, 40 \%$ y $50 \%$ respectivamente. En cuanto a la asistencia a las actividades de EVC, la media se sitúa entre el $74 \%$ y el $85,3 \%$ cuando son grupales en el aula, y entre el $78 \%$ y el $87 \%$ para aquellas a realizar fuera del aula, mientras que en los controles de asistencia no programados la variabilidad es muy amplia, entre el $91,2 \%$ hasta una cota inferior ${ }^{14}$ de $52 \%$.

Desde la semana 7 del curso, la evaluación continua se gamifica ${ }^{15}$ (o ludofica), tal como se muestra en el cronograma, con insignias al alto desempeño, asociadas a las actividades grupales de evaluación y que se materializan en puntuación adicional o excepciones. Así, el 43,3\% de los estudiantes del curso que realizan la actividad gamificada alcanzan la insignia amarilla, que responde a las calificaciones de 8,5 o más, y que responden a una prueba de dificultad baja. Sin embargo, la insignia roja, con una grado de dificultad ligeramente mayor, es obtenida por el $28,8 \%$ de los estudiantes que realizaron la tarea y obtienen una calificación superior a 7 , siendo la insignia verde alcanzada por el 37\% de los estudiantes. Véase Imagen 1 del Anexo.

La fluctuación en notas entre los diversos componentes (prácticas fuera del aula, seminarios en el aula, pruebas de evaluación) para el total de estudiantes tal como se observa en la tabla 5 y el gráfico 12 y en detalle en el gráfico 13 , es reducida.

Indicar que el $22,1 \%$ de los estudiantes liberan la materia sin necesidad de realizar examen final (condicionado a obtener calificaciones en ambas pruebas parciales PEP de 6 o más y tener una evaluación continua de 7 o más), y que se han materializado en medias de 7,613 y 8,15 respectivamente, si bien la primera prueba la liberan el 39,7\% de los estudiantes y la segunda el 30,8\%. Así, en media, los estudiantes objeto del estudio registran una calificación de 6,89 en la Evaluación continua, 5,62 y 5,07 en los pruebas parciales de evaluación (PEP1 Y PEP2 respectivamente) y aquellos que deben realizar prueba final, en media obtienen una calificación de 5,43, siendo de 5,92 la media de la calificación del curso para el total de estudiantes. Al respecto del diferencial en las calificaciones individuales y medias entre pruebas parciales, cabe aportar una reflexión sobre el efecto que aspectos como pueden ser el diseño del sistema de evaluación y tempo-

${ }^{14}$ A pesar de la coordinación en el cronograma de actividades evaluables para todas las materias, disponible desde principio de curso, la cota inferior de asistencia se registrada el día que existe examen parcial liberatorio de otra materia.

${ }^{15}$ Las insignias son comodines para las pruebas de evaluación parciales o finales, que permiten responder un test sin que penalice en caso de error. 
ralización del mismo, o la propia organización académica, especialmente la concentración y el orden de las pruebas de evaluación o exámenes, pueden tener en el rendimiento académico de los estudiantes, tal como evidencian Martín y otros $\left(2003^{16}\right)$, quienes en opinión de los estudiantes encuentran como causas del fracaso escolar el excesivo número de asignaturas simultáneas y la concentración de exámenes. En esta línea argumental, Florido, Jiménez y Navarro (2019) ${ }^{17}$, con un panel de estudiantes universitarios, contrastan que el orden del examen dentro de la convocatoria afecta negativamente al porcentaje de presentados, si bien disponer de al menos un día libre antes del examen no aumenta el porcentaje de alumnos que se presentan.

Al segmentar la población analizada, cabe hacer varias anotaciones: i) los estudiantes varones respectivamente obtienen 6,84 en la EVC, 5,62 en PEP1, 5,43 en PEP2 y 5,79 como calificación final, mientras que las mujeres muestran mejores calificaciones excepto en la EP2, siendo respectivamente $6,96,5,63,4,56$ y 6,11 , si bien los dos rendimientos del curso se corresponden con varones; ii) en cuanto a la nacionalidad, los inscritos con DNI presentan 7,06 en la EVC, 5,74 en PEP1, 5,18 en PEP2 y 5,99 de calificación final, mientras que los que se inscriben con NIE obtienen respectivamente 6,60, $5,40,4,89$ y 6,00 , que muestra un diferencial especialmente en la menor evaluación continua (EVC) y en las pruebas parciales de evaluación (PEP), a pesar de las muchas deficiencias en la comprensión idiomática que muestra el grupo compacto de nacionalidad china.

Tabla 5. Medias elementos de las calificaciones y correlaciones.

\begin{tabular}{|c|c|c|c|c|c|}
\hline & & EVC & PEP1 & PEP2 & $\begin{array}{c}\text { Calificación } \\
\text { final }\end{array}$ \\
\hline \multicolumn{2}{|l|}{ MEDIA } & 6,89 & 5,62 & 5,07 & 5,92 \\
\hline \multicolumn{2}{|c|}{ VARONES (MUJERES) } & $6,84(6,96)$ & $5,62(5,63)$ & $5,43(4,56)$ & $5,79(6,11)$ \\
\hline \multicolumn{2}{|l|}{ DNI (NIE) } & $7,06(6,60)$ & $5,74(5,40)$ & $5,18(4,89)$ & $5,99(6,00)$ \\
\hline Coef. & EVC & & 0,4980 & 0,4979 & 0,4804 \\
\hline Correlación & PEP1 & & & 0,596 & 0,648 \\
\hline Pearson & PEP2 & & & & 0,649 \\
\hline
\end{tabular}

La composición de las calificaciones se refleja en las correlaciones entre los componentes de la calificación final, dado que si bien los seminarios y prácticas son grupales y de ellas se retroalimentan las pruebas parciales de evaluación realizadas individualmente, complementadas con la prueba

${ }^{16}$ Citado en Florido, Jiménez, y Navarro (2019).

17 Resultados académicos de todas las asignaturas en las titulaciones de la Facultad de Economía, Empresa y Turismo de la ULPGC, durante nueve cursos académicos (de 2007-08 a 2015-16), con objeto de controlar los efectos de los cambios normativos y la planificación académica de los exámenes sobre el rendimiento. 
final para aquellos que quieren mejorar su calificación, no liberan materia, por tener menos de 6 en una o las dos pruebas, o las han suspendido. Así, dada las ponderaciones definidas en la calificación final, la correlación (Cf. Pearson) entre la EVC y la calificación final y ( $10 \%$ del total) es de 0,480, y necesariamente mayor entre de las PEP ( $20 \%$ de cada prueba) y la nota final es de 0,648-9 y menor entre los contenidos de la EVC y las pruebas parciales, PEP1 y PEP2, con una correlación uniforme de 0,498.

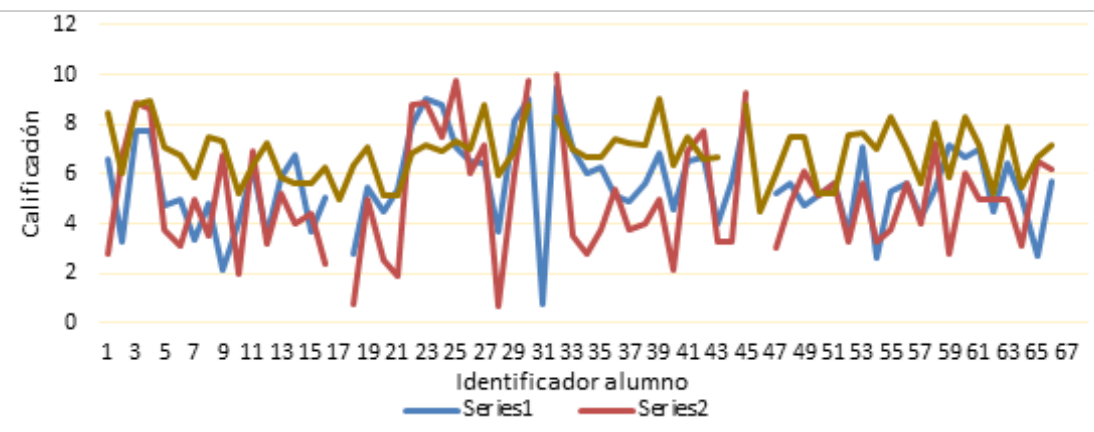

Gráfico 12. Componentes de la calificación final, por alumnos.

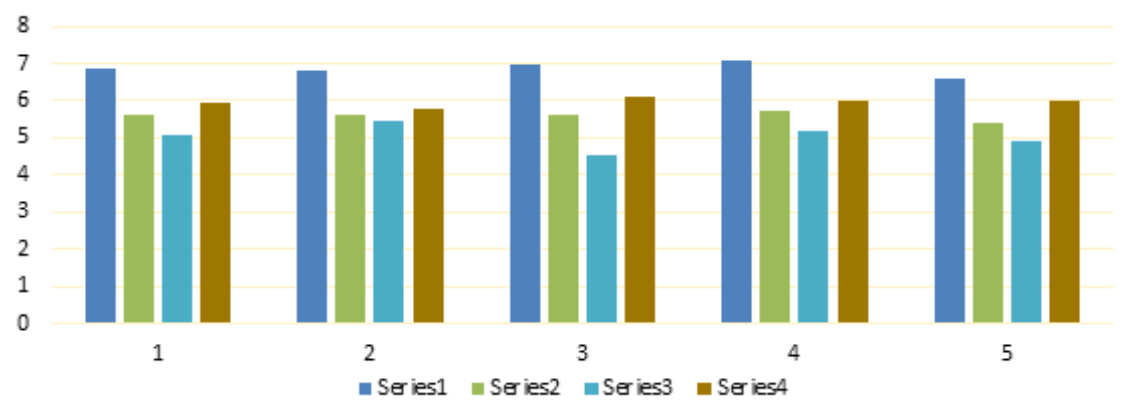

Gráfico 13. Componentes de la calificación final, por segmentaciones.

\subsection{Relación entre calificaciones en AMA con las notas medias del expediente ex ante}

La información personal de la nota media del expediente con la que inician el curso, ex-ante, es de 6,435 para el grupo (información solo disponible para el $73,91 \%$ ), si bien hay claras diferencias por género, siendo de 6,585 para los estudiantes varones y de 6,267 para las mujeres, y que representan un diferencial de $4,823 \%$ más en media favor de los primeros, si bien segmentando el grupo por nacionalidad, las diferencias son mayores, dado que en media la nota media del expediente es 6,691 para los que se inscriben con DNI y 6,005 para quienes se matriculan con NIE, con un diferencial del $11,424 \%$ a favor de los primeros. Sin embargo, observando 
el diferencial y la correlación entre la nota media del expediente al inicio del curso y la calificación de la asignatura objeto del estudio de caso, AMA, las mujeres en la asignatura AMA se han mostrado más uniformes con su expediente que los varones, con 11 puntos porcentuales de diferencia entre sus medias, y de igual forma los estudiantes extranjeros que reproducen casi al $100 \%$ en la asignatura la media de su expediente. Esta información se muestra en la tabla 6 y en los gráficos 14 y 15 .

Tabla 6. Comparativa nota del expediente y la calificación en el curso AMA.

\begin{tabular}{lccccc}
\hline \multicolumn{5}{c}{} & \multicolumn{4}{c}{$\begin{array}{c}\text { Nota } \\
\text { expediente }\end{array}$} & $\begin{array}{c}\text { Calificación } \\
\text { curso AMA }\end{array}$ & $\begin{array}{c}\text { Diferencial } \\
\text { Expediente respecto } \\
\text { curso AMA \% }\end{array}$ & Correlación \\
\hline \multirow{2}{*}{ SEXO } & VARONES & 6,585 & 5,79 & 13,73 & 0,6419 \\
& MUJERES & 6,267 & 6,11 & 2,57 & 0,2289 \\
\multirow{2}{*}{ Nacionalidad } & DNI & 6,691 & 5,99 & 11,70 & 0,4999 \\
TOTAL & NIE & 6,005 & 6,00 & 0,08 & 0,5300 \\
& & 6,435 & 5,92 & 8,70 & 0,5028 \\
\hline
\end{tabular}

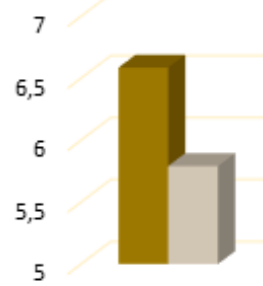

1

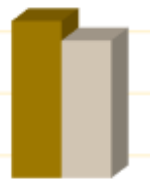

2

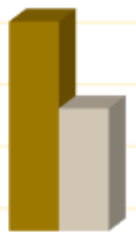

3

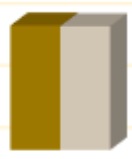

4

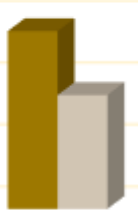

5

- Expediente Calificación AMA

Gráfico 14. Comparativa media nota del expediente y la calificación en el curso AMA, totales.

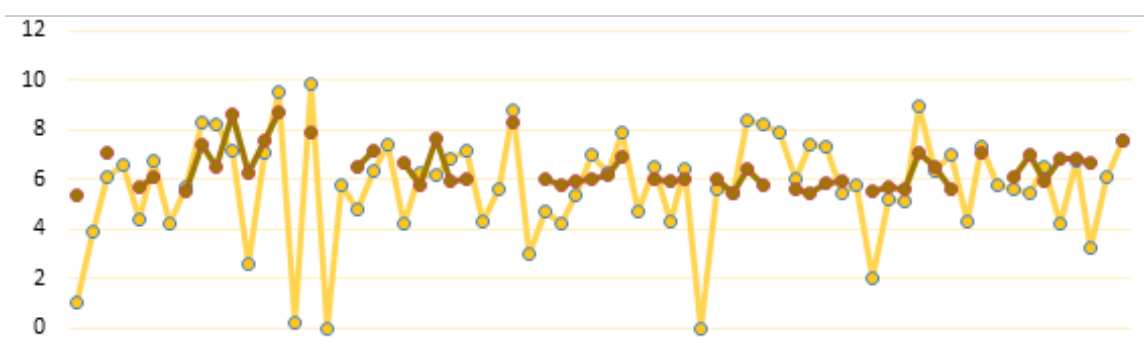

$1 \quad 3 \quad 5 \quad 791113151719212325272931333537394143454749515355575961636567$

$\longrightarrow$-Series $1 \longrightarrow$ Series 2

Gráfico 15. Comparativa media nota del expediente y la calificación en el curso AMA, por alumnos 
La relación entre la actividad registrada de los usos de las herramientas y la nota de expediente y la nota del curso AMA y aporta una última visión del comportamiento del grupo objeto del estudio de caso, que parece no ser dependiente, dado que el coeficiente de correlación entre la brecha digital y la nota del curso es baja, 0,331, y con la nota del expediente únicamente de 0,093. Parece, por tanto, que la intensidad en el uso de las herramientas digitales disponibles para seguir el curso no es intrínseca al rendimiento del estudiante, ni en su expediente ni en el curso de referencia.

Si bien el objetivo de éste estudio de caso es otro, y no permite llevar a cabo un contrastación empírica, sí es oportuno recoger la reflexión final sobre la dependencia de las calificaciones actuales de la nota de expediente, o del rendimiento en materias previas, así como variables inherentes a la organización académica del curso, como ya se ha señalado. Así, la literatura sobre determinantes del rendimiento académico es muy prolija y se ha fijado en indicadores tanto de carácter socio-económico, familiares y afectivas (Tejedor y García-Varcárcel, 2007); de género, estudios previos y sus calificaciones, tipo de acceso a la Universidad, así como las asignaturas aprobadas en el primer curso universitario (por ejemplo en Ibarra y MichaIus, 2010), así como la tipología de las pruebas, el estilo y estrategias para afrontar el estrés, (Ávila-Toscano y otros, 2011); ó en variables específicas de la organización académica (Garbanzo, 2007; Lundberg, 2003; entre otros).

Disponer de información significativa en una muestra más amplia y con un seguimiento continuado de los mismos estudiantes ofrecería una radiografía más clara sobre cómo aspectos socio-personales, de capacidad, de desempeño, de ritmos de trabajo o de estilo, etc. inciden en el rendimiento de los estudiantes.

\section{Discusión de algunos resultados y conclusiones.}

Disponer de la información de los registros de usos, enlazada con las calificaciones de los estudiantes, su comportamiento y su rendimiento previo, así como su valoración sobre la adaptación a los diversos métodos de aprendizaje, permitiría en cursos o intervalos tempranos, definir estrategias de aprendizaje ajustadas a la tipología, que podrían mejorar su rendimiento y compromiso con el programa educativo. Este hecho, si bien hoy parece impensable, será posible muy a corto plazo, en un paso más para ajustar el conocimiento del proceso de enseñanza-aprendizaje a la era digital, dado que las investigaciones de LA interpretan datos educativos mediante aproximaciones cuantitativas sustentadas del comportamiento de los alumnos, pasado, presente o futuro (Amo y Santiago, 2017). 
Lodge y Corrin (2017) inciden en que la analítica del aprendizaje puede aportar información para acometer acciones para reducir ciertas brechas en los rendimientos de la educación, e incluso aplicaciones de la psicología y neurociencia a la educación, con el fin de tener un conocimiento más profundo del comportamiento de los estudiantes en su aprendizaje en entornos educativos reales vs virtuales. Es obvio que no todos los estudiantes interactúan igual con los recursos docentes, ni tienen el mismo patrón y ritmo de usos digitales, como tampoco tienen el mismo estilo de aprendizaje, inteligencia, ni han desarrollado de igual forma el corpus competencial (dependiente tanto de la formación recibida, como del entorno social, familiar y vivencial, así como de las experiencias previas), por lo que afrontar investigaciones que incorporan la explotación de datos de sus registros es ineludible para diseñar (y aplicar) estrategias de aprendizaje adaptables a sus perfiles.

Como conclusiones más directas del estudio de caso, se observa que el perfil de usos de los estudiantes, que dibuja una fuerte brecha digital entre los estudiantes proactivos y los inactivos, debe ser analizado periódicamente y acompasado con el avance del curso, a modo de intervención periódica para recuperar a aquellos estudiantes que evidencian signos de subactividad o descolgamiento del curso, especialmente tras las primeras pruebas de evaluación como medida preventiva.

La dinámica lectiva, reflejada en el cronograma de actividades planificadas durante el curso sigue condicionando el ritmo de accesos y usos virtuales que los estudiantes hacen de las herramientas didácticas, con ciertos desfases dentro de la misma semana, con dispersiones muy altas según tipología, exhibiendo un comportamiento aún tradicional y polarizado su actividad continuada, en contraste con el uso más diversificado del profesor.

Justificar tales divergencias en los roles internos que adquieren los estudiantes en las actividades grupales y el menor interés por determinadas actividades no evaluables, es sólo una visión parcial.

Para la asignatura de referencia, los diferenciales en las calificaciones entre las pruebas parciales y las calificaciones finales, pueden ser explicadas tanto por el grado de dificultad entre las mismas, como por aspectos de desmotivación, o la temporalización del sistema de evaluación continua condicionada a la organización académica, especialmente por la concentración y el orden de las pruebas de evaluación con respecto a otras materias. Más ambicioso sería poder relacionar la información personal de la nota media del expediente, vinculado a la tipología de estudiante según el perfil de uso en registros, así como la segmentación por sexo, o nacionalidad, 
como determinantes del rendimiento del estudiante en el curso, investigación que exigiría disponer de información significativa, que excede los objetivos del trabajo actual.

Estas conclusiones orientan hacia futuras áreas de investigación, y que responden a la líneas que detallan Ferguson, Brasher, Clow y otros (2016), concernientes a los hábitos de los estudiantes durante el proceso de aprendizaje, los desequilibrios en el desarrollo de competencias y habilidades, la interacción en el trabajo en equipo y colaborativo, entre otros.

No cabe dudad de que, recuperando el trabajo de Long y Siemens (2011), el análisis del aprendizaje está permitiendo ver, entre la niebla de la incertidumbre, a las instituciones no sólo cómo asignar eficientemente los recursos y desarrollar ventajas competitivas, sino también disponer de herramientas para mejorar la calidad y el valor de la experiencia de aprendizaje, tanto para el docente como los estudiantes, que abre nuevas oportunidades formativas y áreas de investigación.

\section{Referencias bibliográficas}

Amo, D. y Santiago, R. (2017) Learning Analytics. La narración del aprendizaje a través de los datos. Barcelona: Editorial UOC.

Avila-Toscano, J. H., Hoyos, S. L., González, D. P., y Cabrales, A. (2011). Relación entre ansiedad ante los exámenes, tipos de pruebas y rendimiento académico en estudiantes universitarios. Psicogente, 14, 255-268.

Banihashem S. K., Aliabadi, K., Pourroostae, S., Delaver A, Nili Ahmadabadi, M. (2018). Learning Analytics: A Systematic Literature Review, Interdisciplinary Journal of Virtual Learning in Medical Science, 9(2). https://doi.org/10.5812/ijvlms.63024.

Bates, A.W. (T) (2015). Teaching in a Digital Age: Guidelines for designing teaching and learning for a digital age. University of British Columbia. Recuperado de https://bit.ly/2roovdc

Buckingham S., Ferguson, R. (2012). Social Learning Analytics. Educational Technology \& Society, 15(3), 3-26. Recuperado de https://bit.Iy/2Vja1NB.

Calzadilla, M. E. (2002). Aprendizaje colaborativo y tecnologías de la información y la comunicación. Revista Iberoamericana de Educación, 29(1), 1-10. Recuperado de https://bit.ly/20mLjW1

Campbell, J. P. (2007). Utilizing Student Data within the Course Management System to Determine Undergraduate Student Academic Success: An Exploratory Study, PhD, Purdue University.

Cano, R. (2009). Tutoría universitaria y aprendizaje por competencias ¿Cómo lograrlo? Revista Electrónica Interuniversitaria de Formación del Profesorado, 12(1), 181-204. Recuperado de https://bit.ly/2YfvTHy 
Díaz-Barriga, Á. (2011). Competencias en educación. Corrientes de pensamiento e implicaciones para el currículo y el trabajo en el aula. Revista Iberoamericana de Educación Superior, I/(5), 3-24.

Ferrari, A. (2013) A Framework for Developing and Understanding Digital Competence in Europe. Institute for Prospective Technological Studies. Recuperado de https://bit.ly/23osQ17

Ferguson, R. (2012). Learning analytics: drivers, developments and challenges. International Journal of Technology Enhanced Learning, 4(5/6), 304-317.

Ferguson, R., Brasher A., Clow, D. y otros (2016). Research Evidence on the Use of Learning Analytics - Implications for Education Policy. R. Vuorikari, J. Castaño Muñoz (Eds.). Joint Research Centre Science for Policy Report; EUR 28294 EN; https://doi. org/10.2791/955210.

Ferguson, R. y Clow, D. (2017). Where is the evidence? A call to action for learning analytics. In: LAK '17. Proceedings of the Seventh International Learning Analytics \& Knowledge Conference, ACM International Conference Proceeding Series, pp. 56-65. New York, USA.

Florido, C., Jiménez, J. L., y Navarro, Y. (2019). Normas de permanencia y calendario de exámenes: ¿afectan al rendimiento académico universitario?, Cultura y Educación. https://doi.org/10.1080/11356405.2018.1564571

Garbanzo, G. M. (2007). Factores asociados al rendimiento académico en estudiantes universitarios, una reflexión desde la calidad de la educación superior pública. Revista Educación, 31(1), 43-63.

Guitert, M., Romeu, T. y Pérez, M. (2007). Competencias TIC y trabajo en equipo en entornos virtuales. RUSC. Universities and Knowledge Society Journal, 4(1). Recuperado de https://bit.ly/2H9OQFJ

Gutiérrez, A., Palacios, A. y Torrego, L. (2010). Tribus digitales en las aulas universitarias. Comunicar, 17(34), 173-181.

Harrison, C. y Killion, J. (2007). Ten roles for teachers leaders. Educational Leadership, 65(1), 74-77. Recuperado de https://bit.ly/2CbujQD

Herrero, R. (2014). El papel de las TIC en el aula universitaria para la formación en competencias del alumnado. Pixel-Bit. Revista de Medios y Educación, 45, 173-188. Recuperado de https://bit.ly/2vLp1oV

Ibarra, M. C., y Michalus, J. C. (2010). Análisis del rendimiento académico mediante un modelo logit. Ingeniería Industrial, 9(2), 47-56.

Iglesia, M. C. (2018). Aprendizaje Basado en un Proyecto Docente: Aprendizaje, creatividad, innovación y nuevos roles en la formación de profesorado en la era digital. Revista Complutense de Educación, 29(4), 1253-1278.

Iglesia, M. C. (2006). Varios retos y un único destino en la adaptación de los contenidos de la Teoría Económica al nuevo contexto de enseñanza aprendizaje. El campus virtual como herramienta de apoyo docente. Aplicaciones prácticas de la convergencia europea. Actas Jornadas nacionales de intercambio de experiencias piloto de implantación de metodologías ECTS.

Kaura, P., Singhb, M. y Josanc, G.S. (2015). Classification and prediction based data mining algorithms to predict slow learners in education sector. 3rd International Conference on Recent Trends in Computing (ICRTC-2015).

Leah P. M. y Dawson S., (2010). Mining LMS Data to Develop an 'Early Warning System' for Educators: A Proof of Concept, Computers \& Education, 54(2), 588-599. 
Lodge, J. M., y Corrin, L. (2017). What data and analytics can and do say about effective learning. NpjScience of Learning, 2(1).https://doi.org/10.1038/s41539-017-0006-5

Long, Ph. y Siemens, G. (2011). "Penetrating the Fog: Analytics in Learning and Education". EDUCAUSE Review, 46(5), 30-40.

Lundberg,C. (2003). The influence oftime-limitations, faculty and peer relationships on adult student learning: A causal model. The Journal of Higher Education, 74, 665-688.

Martínez M. E., Arteaga B. y Pérez M. (2017). Description of an experience in university classroom teaching-learning: can the learning of statistics be improved through virtual forums? Conference: ICERI2017, 10th annual International Conference of Education, Research and Innovation. Recuperado de https://bit.ly/2VkUP36

Martín, M. A., Arranz, G., González, M. A., Páramo, R., Alarcia, E., Fernandoz, M. L., y González, M. L. (2003). Análisis del fracaso escolar y desarrollo de acciones de mejora. XI Congreso Universitario de Innovación Educativa en las Enseñanzas Técnicas, Barcelona.

Prensky, M. (2013). Enseñar a nativos digitales (1a. ed). México: SM Ediciones.

Romero, C., y Ventura, S. (2007). Educational data mining: A survey from 1995 to 2005. Expert Systems with Applications, 33(1), 135-146.

Romero, C., Ventura S., y García, E. (2008). Data mining in course management systems: Moodle case study and tutorial. Computers and Education, 51(1), 368-384.

Sampson, D. (2017). Teaching and learning analytics to support teacher inquiry. In 2017 IEEE Global Engineering Education Conference (EDUCON 2017), Apr 25, 2017, Athens, Greece.

Stewart, C. (2017). Learning Analytics: Shifting from theory to practice. Journal on Empowering Teaching Excellence, 1(1), 95-105. https://doi.org/10.15142/T3G63W.

Siemens G. (2013). Learning Analytics: The Emergence of a Discipline, American Behavioral Scientist. Vol 57(10), p.1380-1400, SAGE Publications. Recuperado de https:// bit.ly/2Puq7P8

Siemens, G. y Baker, R. S. J. (2012). Learning analytics and educational data mining: towards communication and collaboration. In Proceedings of the 2nd International Conference on Learning Analytics and Knowledge (LAK '12), Buckingham, Gasevic, and Ferguson (Eds.). ACM, New York, NY, USA, 252-254. https://doi. org/10.1145/2330601.2330661

Siemens, G., Dawson S., y Lynch G. (2013). Improving the Quality of Productivity of the Higher Education Sector: Policy and Strategy for Systems-Level Deployment of Learning Analytics, SoLAR. Recuperadode http://bit.ly/1KNZ9HH

Tejedor, F., y García-Valcárcel, A. (2007). Causas del bajo rendimiento del estudiante universitario (en opinión de los profesores y alumnos). Propuestas de mejora en el marco de EEES. Revista de Educación, 342, 443-473.

Vela-Pérez, M., Hernández-Estrada, A., Tirado-Domínguez, G. y otros (2017). Learning Analytics to classify students according to their activity in moodle. EDULEARN1 7 Proceedings, pp. 1166-1172. Barcelona.

Viñals, A. y Cuenca, J. (2016). El rol del docente en la era digital. Revista Interuniversitaria de Formación del Profesorado, 86(30.2), 103-114. Recuperado de https://bit. Iy/2rq7wKK 


\section{Anexos}

Imagen 1. Evaluación continua y entrega de insignia Amarilla: Reto 1: baja dificultad.

\begin{tabular}{|c|c|c|c|c|c|c|}
\hline & & (t) & $1 / 3$ & \multirow{2}{*}{$\begin{array}{l}\text { AsISTENCIA } \\
25 \text { soptbro }\end{array}$} & \multirow{3}{*}{$\begin{array}{l}\Theta \oplus \\
\text { ASISTENCIA } \\
\text { SDEL II }\end{array}$} & \multirow{3}{*}{$\frac{100 \%}{\text { INSIGNIA }}$} \\
\hline IMENTO & SEMI 3 & PRAC Z & SEMI 4 & & & \\
\hline \multicolumn{5}{|l|}{ b6s54J } & & \\
\hline 327651 & 6 & 5 & & $x$ & $x$ & \\
\hline$\overline{34448 \mathrm{~T}}$ & 7 & 8 & 7,5 & & & \\
\hline 120835 & 7.5 & 10 & 9,5 & $x$ & × & 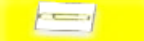 \\
\hline $51694 x$ & 6 & & 6 & $x$ & & \\
\hline $.3561 \Omega$ & 4 & 10 & 83,5 & $x$ & $x$ & $\Leftrightarrow$ \\
\hline $34542 x$ & 7,5 & 10 & 9,5 & $x$ & $x$ & $\Leftrightarrow$ \\
\hline \multicolumn{7}{|l|}{ 38919B } \\
\hline 5783441 & अ & 10 & 8.75 & $x$ & $x$ & $=1$ \\
\hline 319978 & 6 & 10 & & $x$ & N & \\
\hline 4671.37 & $\pi$ & 5 & 7 & $x$ & $x$ & \\
\hline $40460 s$ & 3,5 & & 10 & $x$ & & 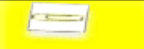 \\
\hline 33066L & s & 10 & 0.75 & $x$ & $x$ & $b=$ \\
\hline $24292 Y$ & 7 & 10 & 7,5 & $x$ & $\lambda$ & \\
\hline 3022222 & $\Leftrightarrow, 5$ & $s, 5$ & 1,5 & $x$ & $x$ & \\
\hline 4916W & 6 & 5 & & $x$ & & \\
\hline Nיבתיבת & 3,5 & 7 & 10 & $x$ & & $=$ \\
\hline
\end{tabular}

Tabla A1. Total registros alumnos AMA 2018_19, y brecha digital.

\begin{tabular}{ccccc}
\hline $\begin{array}{c}\text { Identificador } \\
\text { alumno }\end{array}$ & $\begin{array}{c}\text { Total } \\
\text { Registros }\end{array}$ & $\begin{array}{c}\text { Porcentaje / } \\
\text { Total }\end{array}$ & $\begin{array}{c}\text { Brecha respecto } \\
\text { A12 }\end{array}$ & $\begin{array}{c}\text { Brecha respecto } \\
\text { media }\end{array}$ \\
\hline A12 & 1073 & 4,19 & 0,00 & 193,50 \\
A51 & 1018 & 3,98 & 5,13 & 178,46 \\
A69 & 976 & 3,81 & 9,04 & 166,97 \\
A8 & 755 & 2,95 & 29,64 & 106,52 \\
2A & 714 & 2,79 & 33,46 & 95,30 \\
A17 & 713 & 2,79 & 33,55 & 95,03 \\
A20 & 694 & 2,71 & 35,32 & 89,83 \\
A55 & 681 & 2,66 & 36,53 & 86,28 \\
A16 & 679 & 2,65 & 36,72 & 85,73 \\
A29 & 669 & 2,61 & 37,65 & 82,99 \\
A60 & 648 & 2,53 & 39,61 & 77,25 \\
A28 & 627 & 2,45 & 41,57 & 71,51 \\
A68 & 613 & 2,40 & 42,87 & 67,68 \\
A39 & 541 & 2,11 & 49,58 & 47,98 \\
A56 & 498 & 1,95 & 53,59 & 36,22 \\
A14 & 490 & 1,91 & 54,33 & 34,03 \\
A19 & 476 & 1,86 & 55,64 & 30,20 \\
A18 & 472 & 1,84 & 56,01 & 29,11 \\
A67 & 455 & 1,78 & 57,60 & 24,46 \\
A42 & 450 & 1,76 & 58,06 & 23,09 \\
A45 & 449 & 1,75 & 58,15 & 22,82 \\
A63 & 441 & 1,72 & 58,90 & 20,63 \\
A11 & 405 & 1,58 & 62,26 & 10,78 \\
A36 & 405 & 1,58 & 62,26 & 10,78
\end{tabular}




\begin{tabular}{|c|c|c|c|c|}
\hline $\begin{array}{l}\text { Identificador } \\
\text { alumno }\end{array}$ & $\begin{array}{c}\text { Total } \\
\text { Registros }\end{array}$ & $\begin{array}{c}\text { Porcentaje / } \\
\text { Total }\end{array}$ & $\begin{array}{l}\text { Brecha respecto } \\
\text { A12 }\end{array}$ & $\begin{array}{c}\text { Brecha respecto } \\
\text { media }\end{array}$ \\
\hline A13 & 404 & 1,58 & 62,35 & 10,51 \\
\hline A6 & 403 & 1,57 & 62,44 & 10,23 \\
\hline A58 & 396 & 1,55 & 63,09 & 8,32 \\
\hline A52 & 393 & 1,54 & 63,37 & 7,50 \\
\hline A23 & 380 & 1,48 & 64,59 & 3,94 \\
\hline A27 & 375 & 1,47 & 65,05 & 2,58 \\
\hline A26 & 355 & 1,39 & 66,92 & $-2,90$ \\
\hline A40 & 352 & 1,38 & 67,19 & $-3,72$ \\
\hline A53 & 352 & 1,38 & 67,19 & $-3,72$ \\
\hline A10 & 350 & 1,37 & 67,38 & $-4,26$ \\
\hline A5 & 348 & 1,36 & 67,57 & $-4,81$ \\
\hline A15 & 328 & 1,28 & 69,43 & $-10,28$ \\
\hline A66 & 328 & 1,28 & 69,43 & $-10,28$ \\
\hline A47 & 313 & 1,22 & 70,83 & $-14,38$ \\
\hline A3 & 309 & 1,21 & 71,20 & $-15,48$ \\
\hline A64 & 295 & 1,15 & 72,51 & $-19,31$ \\
\hline A38 & 283 & 1,11 & 73,63 & $-22,59$ \\
\hline A30 & 272 & 1,06 & 74,65 & $-25,60$ \\
\hline A43 & 271 & 1,06 & 74,74 & $-25,87$ \\
\hline $\mathrm{A} 1$ & 258 & 1,01 & 75,96 & $-29,43$ \\
\hline A9 & 257 & 1,00 & 76,05 & $-29,70$ \\
\hline A37 & 252 & 0,98 & 76,51 & $-31,07$ \\
\hline A49 & 251 & 0,98 & 76,61 & $-31,34$ \\
\hline A50 & 246 & 0,96 & 77,07 & $-32,71$ \\
\hline A57 & 244 & 0,95 & 77,26 & $-33,26$ \\
\hline A46 & 236 & 0,92 & 78,01 & $-35,45$ \\
\hline A31 & 231 & 0,90 & 78,47 & $-36,81$ \\
\hline A34 & 220 & 0,86 & 79,50 & $-39,82$ \\
\hline A22 & 214 & 0,84 & 80,06 & $-41,46$ \\
\hline A4 & 210 & 0,82 & 80,43 & $-42,56$ \\
\hline A48 & 210 & 0,82 & 80,43 & $-42,56$ \\
\hline $\mathrm{A} 21$ & 208 & 0,81 & 80,62 & $-43,10$ \\
\hline A35 & 182 & 0,71 & 83,04 & $-50,22$ \\
\hline A25 & 152 & 0,59 & 85,83 & $-58,42$ \\
\hline A65 & 152 & 0,59 & 85,83 & $-58,42$ \\
\hline A32 & 135 & 0,53 & 87,42 & $-63,07$ \\
\hline A33 & 130 & 0,51 & 87,88 & $-64,44$ \\
\hline A44 & 76 & 0,30 & 92,92 & $-79,21$ \\
\hline A59 & 76 & 0,30 & 92,92 & $-79,21$ \\
\hline A41 & 68 & 0,27 & 93,66 & $-81,40$ \\
\hline A62 & 51 & 0,20 & 95,25 & $-86,05$ \\
\hline A7 & 36 & 0,14 & 96,64 & $-90,15$ \\
\hline A24 & 34 & 0,13 & 96,83 & $-90,70$ \\
\hline A61 & 9 & 0,04 & 99,16 & $-97,54$ \\
\hline $\mathrm{AO}$ & 4 & 0,02 & 99,63 & $-98,91$ \\
\hline A54 & 0 & 0,00 & 100,00 & $-100,00$ \\
\hline
\end{tabular}


Tabla A2. Porcentaje de registros por alumnos y semanas, AMA 2018_19.

Selección 4 semanas iniciales de curso y 4 finales.

\begin{tabular}{|c|c|c|c|c|c|c|c|c|}
\hline $\begin{array}{l}\text { Identifica- } \\
\text { dor }\end{array}$ & $\begin{array}{c}\text { Sem } \\
1\end{array}$ & Sem 2 & Sem 3 & Sem 4 & $\begin{array}{c}\text { Sem } \\
15\end{array}$ & $\begin{array}{c}\text { Sem } \\
16\end{array}$ & $\begin{array}{c}\text { Sem } \\
17\end{array}$ & $\begin{array}{c}\text { Sem } \\
18\end{array}$ \\
\hline 0 & 0,00 & 0,00 & 0,00 & 0,00 & 0,00 & 0,00 & 0,00 & 0,06 \\
\hline 1 & 1,11 & 1,00 & 0,45 & 0,39 & 5,10 & 0,56 & 1,08 & 0,49 \\
\hline 2 & 1,25 & 0,82 & 2,29 & 1,70 & 2,55 & 1,67 & 1,80 & 4,93 \\
\hline 3 & 1,39 & 0,47 & 0,55 & 0,52 & 0,51 & 0,28 & 3,77 & 1,29 \\
\hline 4 & 0,00 & 0,18 & 0,55 & 1,05 & 3,57 & 1,67 & 6,46 & 1,23 \\
\hline 5 & 0,00 & 0,00 & 1,19 & 4,84 & 1,02 & 0,00 & 0,00 & 4,80 \\
\hline 6 & 0,00 & 1,53 & 0,94 & 1,05 & 0,00 & 0,00 & 0,54 & 0,80 \\
\hline 7 & 0,00 & 0,00 & 0,00 & 0,00 & 0,00 & 0,00 & 0,00 & 0,18 \\
\hline 8 & 11,00 & 0,00 & 1,99 & 1,83 & 1,53 & 0,56 & 1,80 & 2,65 \\
\hline 9 & 0,00 & 0,12 & 0,40 & 1,31 & 4,08 & 0,28 & 1,26 & 5,23 \\
\hline 10 & 0,00 & 0,76 & 0,84 & 2,62 & 3,06 & 0,28 & 1,26 & 2,03 \\
\hline 11 & 1,81 & 0,65 & 2,34 & 1,57 & 2,04 & 0,00 & 0,54 & 3,08 \\
\hline 12 & 3,48 & 5,40 & 1,74 & 4,58 & 1,02 & 0,56 & 9,87 & 7,94 \\
\hline 13 & 11,98 & 2,41 & 1,34 & 0,52 & 1,02 & 0,56 & 0,00 & 0,43 \\
\hline 14 & 0,70 & 0,06 & 0,50 & 2,09 & 1,53 & 14,21 & 1,80 & 0,68 \\
\hline 15 & 0,00 & 0,65 & 0,94 & 1,44 & 2,55 & 0,00 & 1,44 & 4,13 \\
\hline 16 & 0,97 & 7,57 & 7,31 & 1,31 & 0,00 & 0,00 & 1,80 & 4,56 \\
\hline 17 & 1,67 & 0,88 & 2,88 & 3,93 & 7,65 & 2,51 & 0,54 & 1,60 \\
\hline 18 & 3,48 & 4,40 & 0,40 & 0,00 & 0,00 & 0,00 & 2,33 & 1,72 \\
\hline 19 & 0,00 & 0,82 & 1,39 & 1,05 & 3,57 & 2,23 & 2,15 & 0,43 \\
\hline 20 & 0,70 & 2,58 & 2,04 & 6,81 & 1,53 & 0,28 & 0,36 & 1,11 \\
\hline 21 & 0,00 & 0,06 & 1,54 & 0,79 & 1,02 & 13,93 & 0,00 & 1,48 \\
\hline 22 & 0,00 & 0,23 & 0,15 & 0,65 & 0,00 & 0,00 & 0,18 & 1,79 \\
\hline 23 & 0,84 & 0,65 & 0,50 & 0,92 & 1,02 & 0,00 & 0,18 & 7,08 \\
\hline 24 & 0,14 & 0,41 & 0,15 & 0,00 & 0,00 & 0,00 & 0,18 & 0,00 \\
\hline 25 & 0,00 & 3,58 & 0,30 & 0,79 & 0,51 & 0,00 & 0,36 & 0,99 \\
\hline 26 & 1,39 & 8,04 & 0,20 & 0,52 & 0,51 & 0,00 & 3,05 & 0,99 \\
\hline 27 & 0,00 & 2,05 & 6,01 & 1,18 & 0,51 & 0,00 & 5,75 & 5,67 \\
\hline 28 & 12,12 & 1,17 & 1,09 & 1,05 & 5,61 & 1,11 & 3,05 & 0,80 \\
\hline 29 & 1,25 & 0,23 & 2,73 & 1,44 & 4,59 & 1,95 & 1,26 & 1,72 \\
\hline 30 & 0,00 & 0,41 & 0,94 & 0,00 & 0,00 & 0,00 & 1,80 & 2,28 \\
\hline 31 & 0,00 & 0,06 & 1,14 & 1,18 & 0,00 & 0,28 & 0,90 & 0,62 \\
\hline 32 & 0,00 & 0,18 & 0,60 & 1,05 & 3,57 & 0,00 & 1,26 & 0,18 \\
\hline 33 & 0,00 & 0,29 & 0,89 & 0,00 & 0,00 & 1,67 & 0,00 & 0,74 \\
\hline 34 & 0,56 & 0,00 & 0,84 & 3,40 & 0,51 & 0,28 & 0,00 & 0,49 \\
\hline 35 & 0,00 & 2,58 & 0,50 & 3,66 & 0,51 & 0,00 & 1,62 & 0,18 \\
\hline 36 & 2,23 & 2,41 & 0,99 & 1,05 & 0,00 & 0,00 & 0,36 & 1,91 \\
\hline 37 & 0,00 & 0,59 & 0,20 & 0,00 & 0,00 & 0,28 & 0,18 & 0,18 \\
\hline 38 & 0,00 & 1,88 & 2,24 & 2,09 & 0,51 & 0,00 & 0,00 & 2,03 \\
\hline 39 & 0,70 & 0,12 & 0,55 & 1,31 & 1,02 & 14,21 & 0,18 & 1,35 \\
\hline 40 & 0,00 & 1,00 & 0,50 & 1,70 & 0,51 & 0,00 & 0,36 & 7,20 \\
\hline 41 & 0,84 & 0,12 & 0,05 & 0,13 & 0,00 & 0,00 & 1,08 & 1,42 \\
\hline
\end{tabular}




\begin{tabular}{|c|c|c|c|c|c|c|c|c|}
\hline $\begin{array}{c}\text { Identifica- } \\
\text { dor }\end{array}$ & $\begin{array}{c}\text { Sem } \\
1\end{array}$ & Sem 2 & Sem 3 & Sem 4 & $\begin{array}{c}\text { Sem } \\
15\end{array}$ & $\begin{array}{c}\text { Sem } \\
16\end{array}$ & $\begin{array}{c}\text { Sem } \\
17\end{array}$ & $\begin{array}{c}\text { Sem } \\
18\end{array}$ \\
\hline 42 & 1,53 & 7,45 & 4,03 & 0,65 & 0,51 & 0,00 & 4,49 & 1,23 \\
\hline 43 & 0,00 & 0,65 & 2,34 & 0,52 & 5,10 & 0,56 & 4,85 & 4,68 \\
\hline 44 & 1,11 & 0,00 & 0,80 & 0,00 & 0,00 & 0,00 & 0,00 & 0,49 \\
\hline 45 & 0,00 & 0,70 & 0,70 & 1,44 & 1,02 & 0,00 & 0,18 & 1,23 \\
\hline 46 & 11,00 & 0,00 & 1,39 & 0,39 & 2,04 & 0,56 & 1,80 & 0,25 \\
\hline 47 & 1,25 & 0,00 & 0,15 & 0,00 & 0,00 & 1,67 & 0,00 & 0,37 \\
\hline 48 & 0,00 & 0,06 & 0,40 & 0,00 & 2,04 & 0,28 & 1,97 & 1,17 \\
\hline 49 & 0,00 & 0,18 & 0,89 & 1,31 & 2,55 & 0,28 & 1,62 & 0,49 \\
\hline 50 & 0,00 & 0,41 & 1,34 & 1,05 & 3,57 & 1,67 & 0,18 & 2,16 \\
\hline 51 & 0,56 & 1,53 & 3,78 & 9,16 & 1,02 & 0,28 & 2,69 & 3,76 \\
\hline 52 & 2,23 & 3,99 & 0,35 & 1,31 & 0,00 & 0,00 & 0,00 & 1,48 \\
\hline 53 & 0,00 & 0,59 & 0,15 & 0,00 & 0,00 & 0,28 & 0,72 & 3,76 \\
\hline 54 & 0,00 & 0,00 & 0,00 & 0,00 & 0,00 & 0,00 & 0,00 & 0,00 \\
\hline 55 & 2,09 & 2,99 & 1,89 & 4,19 & 1,53 & 7,80 & 5,21 & 2,83 \\
\hline 56 & 0,00 & 0,59 & 1,54 & 1,31 & 1,53 & 7,80 & 3,95 & 7,57 \\
\hline 57 & 0,97 & 2,00 & 0,30 & 0,13 & 0,00 & 0,00 & 0,72 & 3,20 \\
\hline 58 & 6,41 & 5,46 & 4,42 & 0,52 & 0,51 & 0,56 & 0,00 & 0,37 \\
\hline 59 & 5,01 & 0,00 & 1,34 & 0,00 & 0,00 & 0,00 & 0,54 & 0,12 \\
\hline 60 & 1,95 & 0,88 & 3,08 & 1,31 & 7,14 & 0,56 & 3,59 & 2,22 \\
\hline 61 & 0,00 & 0,00 & 0,00 & 0,13 & 0,00 & 0,00 & 0,00 & 0,00 \\
\hline 62 & 0,00 & 0,00 & 0,10 & 0,52 & 0,51 & 0,00 & 0,00 & 0,06 \\
\hline 63 & 1,25 & 0,41 & 1,54 & 0,26 & 2,04 & 1,67 & 0,18 & 2,59 \\
\hline 64 & 0,00 & 0,35 & 0,89 & 0,79 & 0,00 & 0,00 & 1,26 & 0,92 \\
\hline 65 & 0,00 & 0,06 & 0,25 & 3,40 & 0,51 & 0,28 & 1,08 & 0,49 \\
\hline 66 & 0,56 & 2,76 & 0,55 & 1,31 & 3,57 & 1,95 & 2,15 & 0,18 \\
\hline 67 & 2,23 & 2,88 & 0,89 & 3,80 & 0,51 & 0,00 & 0,54 & 1,72 \\
\hline 68 & 0,70 & 1,88 & 1,79 & 3,14 & 0,51 & 10,58 & 0,00 & 0,43 \\
\hline 69 & 0,97 & 7,86 & 12,92 & 1,83 & 1,02 & 3,90 & 1,80 & 7,14 \\
\hline Total & 100 & 100 & 100 & 100 & 100 & 100 & 100 & 100 \\
\hline
\end{tabular}

